\title{
Fabrication and electrochemical response of pristine graphene ultramicroelectrodes
}

\section{DOI:}

DOI: 10.1016/j.carbon.2021.02.078

\section{Document Version}

Accepted author manuscript

Link to publication record in Manchester Research Explorer

\section{Citation for published version (APA):}

Goodwin, S., Coldrick, Z., Heeg, S., Grieve, B., Vijayaraghavan, A., \& Hill, E. W. (2021). Fabrication and electrochemical response of pristine graphene ultramicroelectrodes. Carbon, 177, 207-215. https://doi.org/DOI: 10.1016/j.carbon.2021.02.078

\section{Published in:}

Carbon

\section{Citing this paper}

Please note that where the full-text provided on Manchester Research Explorer is the Author Accepted Manuscript or Proof version this may differ from the final Published version. If citing, it is advised that you check and use the publisher's definitive version.

\section{General rights}

Copyright and moral rights for the publications made accessible in the Research Explorer are retained by the authors and/or other copyright owners and it is a condition of accessing publications that users recognise and abide by the legal requirements associated with these rights.

\section{Takedown policy}

If you believe that this document breaches copyright please refer to the University of Manchester's Takedown Procedures [http://man.ac.uk/04Y6Bo] or contact uml.scholarlycommunications@manchester.ac.uk providing relevant details, so we can investigate your claim.

\section{OPEN ACCESS}




\title{
Fabrication and electrochemical response of pristine graphene ultramicroelectrodes
}

Stefan Goodwin ${ }^{1}$, Zachary Coldrick ${ }^{1}$, Sebastian $\mathrm{Heeg}^{2,3, \dagger}$, Bruce Grieve ${ }^{1}$, Aravind Vijayaraghavan ${ }^{2,3, *}$, Ernie W Hill ${ }^{3,4}$

${ }^{1}$ Department of Electrical and Electronic Engineering, The University of Manchester, Oxford Road, Manchester M13 9PL, UK

${ }^{2}$ Department of Materials, The University of Manchester, Oxford Road, Manchester M13 9PL, UK

${ }^{3}$ National Graphene Institute, The University of Manchester, Oxford Road, Manchester M13 9PL, UK

${ }^{4}$ Department of Computer Science, The University of Manchester, Oxford Road, Manchester, M13 9PL, UK.

${ }^{\dagger}$ Present address: Department of Physics, Freie Universität Berlin, 14195 Berlin, Germany

\begin{abstract}
Ultramicroelectrodes (UMEs) are electrochemical working electrodes with at least one dimension less than $25 \mu \mathrm{m}$. Here, we demonstrate a method to fabricate pristine (contamination and doping-free) monolayer graphene UMEs and characterise its electrochemical properties. Graphene is particularly sensitive to both contamination from device fabrication and doping from the substrate, and these effects are detrimental to accurate measurements of fundamental electrochemical properties as well as electrochemical electrode applications. We demonstrate a UME design and fabrication steps which ensure that the electro-active surface of the graphene never comes into contact with contaminants such as process polymers and solvents, has a well-defined area due to self-aligned fabrication, and we shield the converse side of the graphene monolayer with the highly inert dielectric hexagonal boron nitride (h$\mathrm{BN})$. Cyclic voltammetry demonstrates the expected steady-state behaviour for a UME in the hemispherical diffusion regime. The reduction of $\mathrm{IrCl}_{6}{ }^{2-}$ in weak $\mathrm{KCl}$ electrolytes was employed to investigate the electron transfer characteristics of the pristine graphene UMEs and the reproducibility of the measurements. Values of the standard rate constant, $k_{0}$ and the transfer coefficient, $\alpha$ were measured to be $3.04 \pm 0.78 \times 10^{-3} \mathrm{~cm} \cdot \mathrm{s}^{-1}$ and $0.272 \pm 0.024$ respectively. These values are consistent for monolayer graphene with reduced charge doping from contaminants and the substrate. This is further confirmed by Raman spectroscopy measurements of the graphene UME before and after the electrochemical measurements.
\end{abstract}

Keywords: Graphene, electrochemistry, ultramicroelectrode, boron nitride, contamination, doping.

* Corresponding author: Tel: +44 (0)161275 0136. E-mail: aravind@ manchester.ac.uk 


\section{Introduction}

Since the first graphene electronic device was demonstrated in 2004 [1], sensors have emerged as the dominant application field for such devices, [2] since many of the properties of graphene are particularly suited to various sensing technologies. The electronic properties of graphene are highly sensitive to its environment, owing to its unique electronic structure and the fact that every carbon atom of graphene is exposed to the environment, twice over. Of the different kinds of sensors, electrochemical sensors hold the greatest potential for biological and medical applications [3] due to the ubiquitous presence of water and the constant need for improved monitoring in fields ranging from healthcare to agriculture. Ultramicroelectrodes (UMEs) are electrochemical working electrodes where the size of the active sensing electrode is of micron scale [4]; a working definition of UME calls for at least one dimension to be less than $25 \mu \mathrm{m}$. Faster double-layer charging, reduced ohmic loss, and high mass-transport rates have allowed UMEs to revolutionize thermodynamic, kinetic, and electroanalytical measurements. UMEs are favourable for applications requiring high spatial resolution or interfacing with objects such as individual cells. Electrochemical electrodes can be reduced to micron size using semiconductor lithography techniques [5] or using epoxy resin or similar polymers as a mask material [6].

There are three key challenges in the fabrication of graphene UME: undesired and uncontrolled doping arising from (i) contamination of the graphene from transfer polymer and (ii) the substrate that the graphene is interfaced to, and (iii) poorly defined electrode area arising from limitations in device fabrication and graphene surface contaminations.

Investigations of the electrochemical properties of graphene have suffered from challenges such as variations in the quality of the graphene material, contamination and defects arising from fabrication steps, [7] and fabrication process complexities in seeking to overcome these challenges. Due to the extreme sensitivity and affinity of graphene to its immediate environment, any substances that graphene comes into contact with during growth, transfer or device fabrication leads to uncontrollable surface modifications and unpredictable changes in measured device characteristics [8]. Polymers commonly used in micro-fabrication leave persistent contamination which cannot be removed without damaging the graphene $[9,10]$. For electrochemical measurements in particular, surface residues will alter the electron transfer characteristics and reduce the available area for chemical interactions at the grapheneelectrolyte interface [11]. Annealing devices at a high temperature in $\mathrm{H}_{2} / \mathrm{Ar}$ atmosphere can partially remove the adsorbed residues, however this process can remove intended chemical functionalities so is not always ideal [12]. Graphene devices cannot be cleaned by traditional semiconductor methods such as reactive plasma [13], UV/Ozone [14], or chemical etches [15] as they will all result in chemical interactions that will damage the graphene. Due to this, methods that avoid exposure to polymers during device fabrication are ideal and increasingly being explored for various devices [16-18]. 
The dielectric layer, which is often the underlying substrate against which graphene is placed and structured, significantly influences the performance of the resulting device [19, 20]. These effects are primarily a result of dielectric surface roughness and inhomogeneous trapped charges. Surface roughness causes carrier scattering at topographical changes and corrugations, resulting in reduced charge carrier mean free path and mobility [21]. A bigger effect stems from charge inhomogeneity, caused by charged impurities trapped on or near the surface of several dielectric materials, in particular $\mathrm{SiO}_{2}$. These charged impurities lead to the local accumulation of mirror charge carriers in a graphene sheet placed against the dielectric, resulting in carrier density fluctuations known as electron-hole puddles [22]. Carrier concentration plays an important role in electron transfer chemistry, with areas of higher charge concentration providing a higher density of electrons or holes to participate in chemical reactions. Therefore, dielectrics that induce a smaller amount of charge fluctuation can achieve lower chemical reactivity [23]. Hydrophobic coatings on a dielectric surface can help reduce the adsorption of charged molecules and provide electronic screening of charged impurities; hence, graphene interfaced against these surfaces has increased charge carrier mobility and lower chemical reactivity $[24,25]$. Furthermore, the effect of the dielectric-induced charge inhomogeneity should be considered while interpreting results that indicate that the electrochemical electron transfer rate decreases with increasing number of graphene layers [26], as the opposite should be true due to the effective density of electronic states (DoS) increasing with extra layers. Due to its atomic flatness and lack of charge traps, hexagonal boron nitride (h-BN) has become a favoured dielectric substrate for graphene electronic devices where preservation of the intrinsic electronic properties of graphene is desired [27].

Lastly, electrochemical UMEs require a very well-defined area, since the currents due to electron transfer are directly proportional to the electrode area. Previous studies of graphene electrochemical electrodes have struggled to produce a well-defined graphene area with precise boundaries and not including defective regions of the graphene flake or adding significant contamination [28, 29]. Surface contaminants described above have the additional effect of reducing the effective graphene electrode area [26]. These all lead to uncertainties in the calculation of electrochemical parameters from measured currents due to uncertainties in the actual electrode area participating in the process.

Here we describe a lithography-free fabrication method for micron-scale graphene electrochemical devices which overcomes the challenges described above. The method results in a self-aligned and welldefined active area, interfaced with a highly inert dielectric, and without ever exposing the active surface to polymer contamination. The devices presented were fabricated using single crystal mechanically exfoliated monolayer graphene samples interfaced against mechanically exfoliated h-BN dielectric layers. The kinetic parameters of electrochemical heterogeneous electron transfer on this graphene were then investigated and compared to previous studies on graphene in other configurations. The level of doping in the graphene before and after electrochemical electron transfer is evaluated using Raman spectroscopy. 


\section{Experimental}

\subsection{UME device concept and design}

A traditionally fabricated graphene device typically has a graphene flake over an underlying dielectric substrate, with metal electrodes fabricated on the top surface of the graphene using lithographic processes involving polymer resists [1]. The graphene itself, whether mechanically exfoliated or grown by chemical vapour deposition, is transferred from the parent substrate to the final substrate using transfer materials that adhere to the top surface of the graphene, leaving the bottom surface free of polymer residues. In this work, we propose and develop a device design (Figure 1) wherein the uncontaminated bottom graphene surface is exposed by means of a hole in the underlying substrate to form the UME. This substrate hole, which can be pre-defined by lithography in a rigid material, has the added advantage of precisely defining the area of the graphene UME participating in the electrochemical process, and this UME area is not reduced in any unpredictable manner by defects or contamination. The other side of the graphene flake is interfaced against h-BN, the best-known dielectric material for graphene, prior to any exposure to the transfer polymer. This preserves the intrinsic graphene electronic properties and excludes dielectric trapped charges or scattering sites. Lastly, the process of defining the substrate hole inherently forms a fluid reservoir in the underlying substrate, which can be interfaced with microfluidics and micro-scale counter and reference electrodes to form a highly miniaturised UME requiring very small analyte volumes.

\subsection{UME device fabrication}

A monolayer graphene sample was produced via mechanical exfoliation on the $\mathrm{SiO}_{2}$ surface of a $\mathrm{Si}$ substrate, as previously described [2]. A mechanically exfoliated, $\sim 80 \mathrm{~nm}$ thickness h-BN flake was then produced on a second $\mathrm{SiO}_{2} / \mathrm{Si}$ substrate using the same method (further detail in Supporting Information Figure 2). The h-BN flake was then transferred on top of the previously prepared graphene layer, using the 'wet' polymer transfer method with polymethylmethacrylate (PMMA) as the transfer polymer [30]. The h-BN flake needs to fully cover the area of the graphene intended to act as the active electrochemical electrode, but be small enough that an area outside this is available for making electrical contact to the graphene with a metal, shown schematically in Figure 1(a).

A Si wafer piece with $100 \mathrm{~nm}$ thickness silicon nitride $\left(\mathrm{SiN}_{\mathrm{x}}\right)$ coating on both surfaces was selected to act as the UME body. A 100x100 $\mu \mathrm{m}^{2}, 100 \mathrm{~nm}$ thick, suspended $\operatorname{SiN}_{\mathrm{x}}$ membrane was created using optical lithography followed by reactive ion etching (RIE) and wet $\mathrm{KOH}$ etching, following a procedure described in previous work [31] (further detail in Supporting Information Figure 1). A hole was then created using RIE in the $\mathrm{SiN}_{\mathrm{x}}$ membrane, with the area of the hole defining what would be the electrochemically active area on the graphene UME. For this study circular holes of 10 and $15 \mu \mathrm{m}$ diameter were used, ensuring the devices were small enough to be within the UME regime but large 
(a)

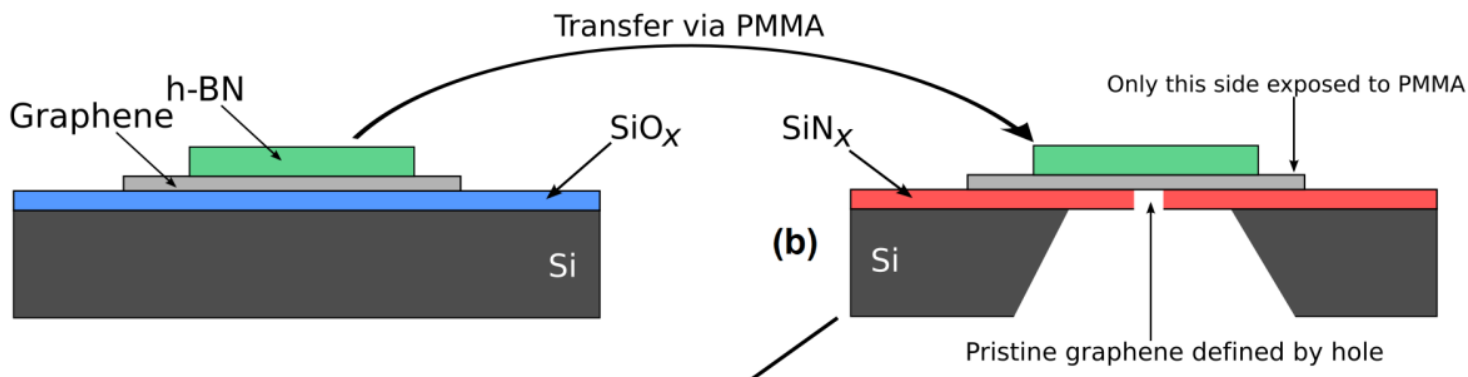

(c)

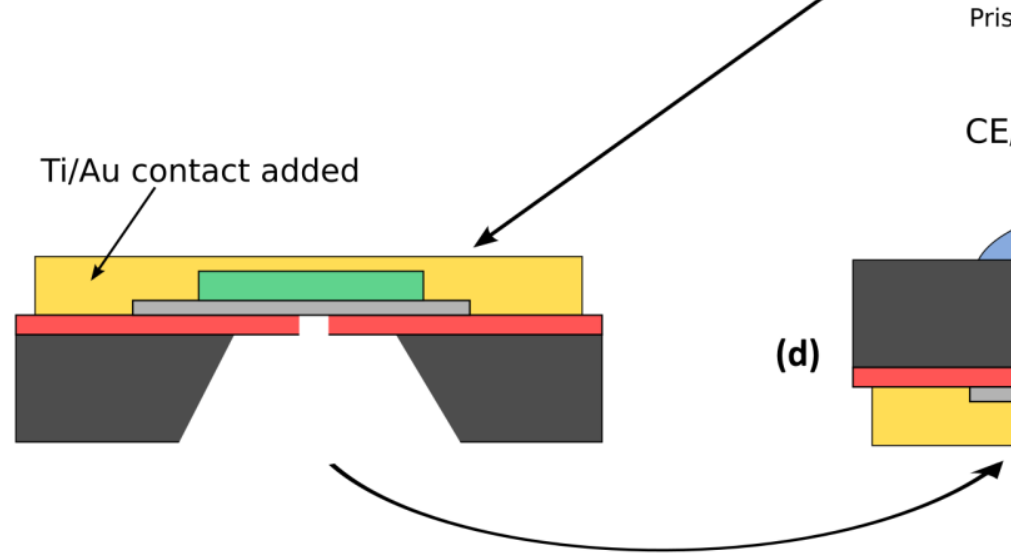

Turn upside down and add electrolyte
Pristine graphene defined by hole

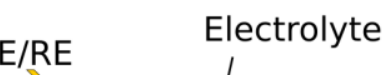

* Hole dimensions -

Depth: $100 \mathrm{~nm}$

Diameter: $10-15 \mu \mathrm{m}$

Figure 1 -Schematic outlining the main steps for the assembly of the graphene on h-BN electrode, where WE, CE and RE are the working, counter and reference electrodes respectively.

enough to give easily measurable currents. The wafers were then exposed to vapour phase HMDS to create a hydrophobic surface.

The graphene/h-BN structure previously prepared was then transferred on to the upper surface of the $\mathrm{SiN}_{\mathrm{x}}$ membrane, ensuring it entirely covers the fabricated hole as shown schematically in Figure 1(b). As the hole defines the electrochemically active area, the graphene needed to be positioned so that there were no obvious microscopic or macroscopic defects in this region, with the optical dark-field (DF) images of the flake used to aid this process. The completed stack represented in Figure $1 \mathrm{~b}$ is seen in the optical micrographs in Figure 2, in top and bottom view, outlining the flakes and $\mathrm{SiN}_{\mathrm{x}}$ window. A single $\mathrm{Ti}(5 \mathrm{~nm}) / \mathrm{Au}(100 \mathrm{~nm})$ metallic contact was then deposited over the graphene/h-BN stack by electron beam evaporation. No lithography was needed and the surface of the wafer could be uniformly covered in metal, since the h-BN flake acts as a self-aligned mask to ensure that no metal is deposited directly on the graphene in the UME area defined by the hole. In order to ensure that there is no contact between the metal and the silicon underneath the $\mathrm{SiN}_{\mathrm{x}}$ surface, the edges of the wafer were protected from the evaporated metal using polyimide (Kapton) tape window as a mask. After deposition the tape could be peeled away, leaving metal only in the central area covering the h-BN-graphene flakes, as shown schematically in Figure 1(c). The completed chip was mounted in a modified chip carrier as described in the supplementary information.

This fabrication method creates a structure where the electrochemically active graphene surface is not exposed to polymer contaminants, and can be further developed to include lithography steps to etch the 


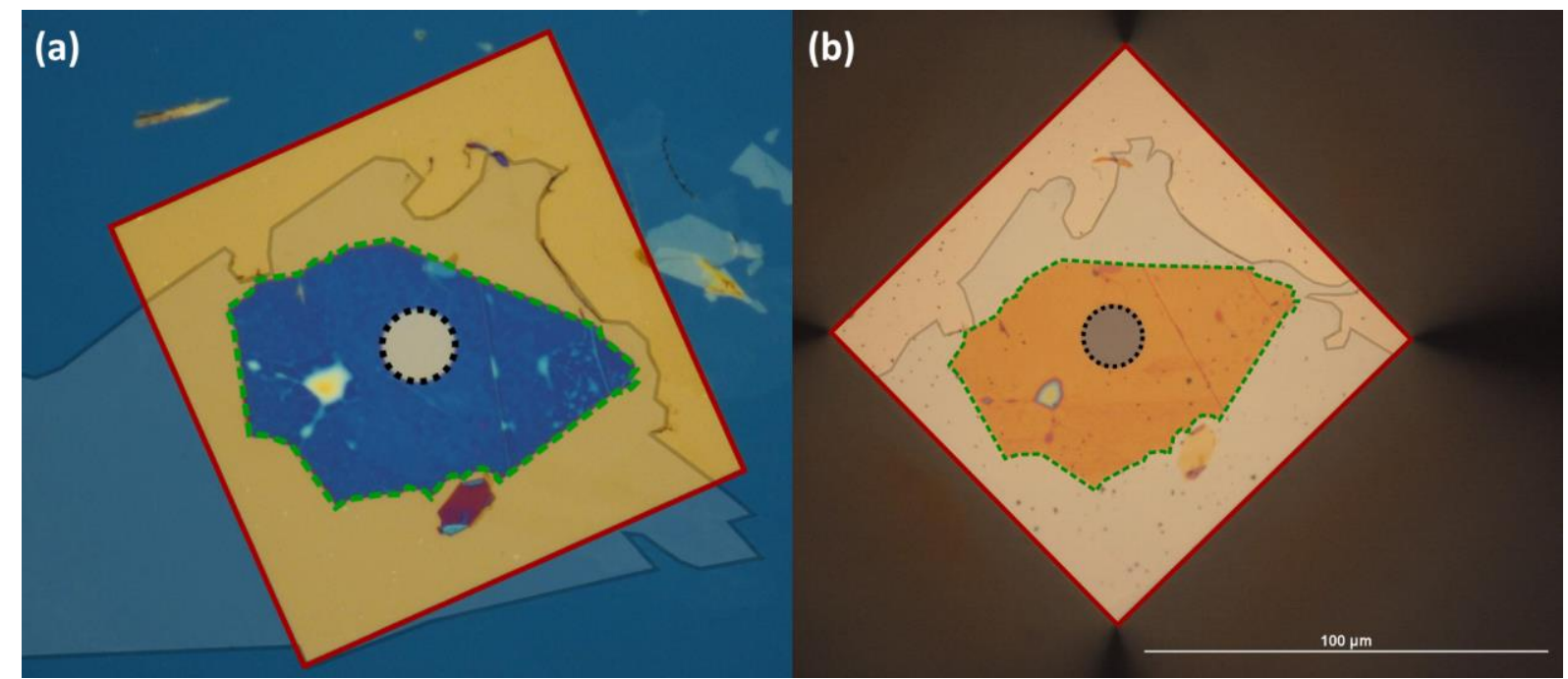

Figure 2 - (a) Top and (b) bottom view images of the Si/SiNx wafer with the finished device. The constituent parts are highlighted, with the grey shaded area highlighting the graphene flake, the green dotted line outlining the $h$-BN substrate, the black dotted line outlining the hole in the silicon nitride and therefore the electrochemically active area of the graphene. The red line is the edge of the silicon nitride 'window'.

graphene into a pre-defined shape or to include multiple electronic contacts (demonstrated in supporting information Figure S3(b), all without polymer deposition on the active electrochemical area, and is therefore a truly flexible method that could be used in a variety of graphene sensing device structures. It is possible to use the dry 'stamp' transfer technique [32] to further limit the ionic and solvent contamination of the graphene during fabrication, however, this paper focusses on results using the wet transfer method due to greater control of exfoliated flake surface areas.

The devices were measured on a custom electrochemical measurement system using a borosilicate glass pipette to position a drop of electrolyte on to the active surface of the graphene electrode within the pyramidal cavity in the silicon substrate, as shown in Figure 1(d) and supporting information Figure S5. Two wires within the pipette functioned as $\mathrm{Ag} / \mathrm{AgCl}$ reference electrode (RE) and $\mathrm{Pt}$ counter electrode (CE), with the graphene electrode surface acting as the working electrode (WE). This configuration lends itself more broadly to being integrated into well-defined microfluidic channels interfaced with the graphene UME.

\section{Results}

Redox measurements were made using $3 \mathrm{mM}$ of the ammonium salt of $\mathrm{IrCl}_{6}^{2-}$ (ammonium hexachloroiridate (IV), Sigma Aldrich) in a $0.15 \mathrm{M} \mathrm{KCl}$ electrolyte. This concentration of supporting electrolyte was chosen to demonstrate use in biologically relevant electro-chemistries, in particular to ensure that evaporation from the small analyte droplet is not excessive. This concentration is sufficient to ensure that the Debye length is negligible compared to the dimension of the diffusion layer, ensuring 
the redox couple only experiences diffusive transport and charge migration is negligible [33] (further details in supporting information S2). This outer sphere redox mediator undergoes a reduction to $\mathrm{IrCl}_{6}{ }^{3-}$
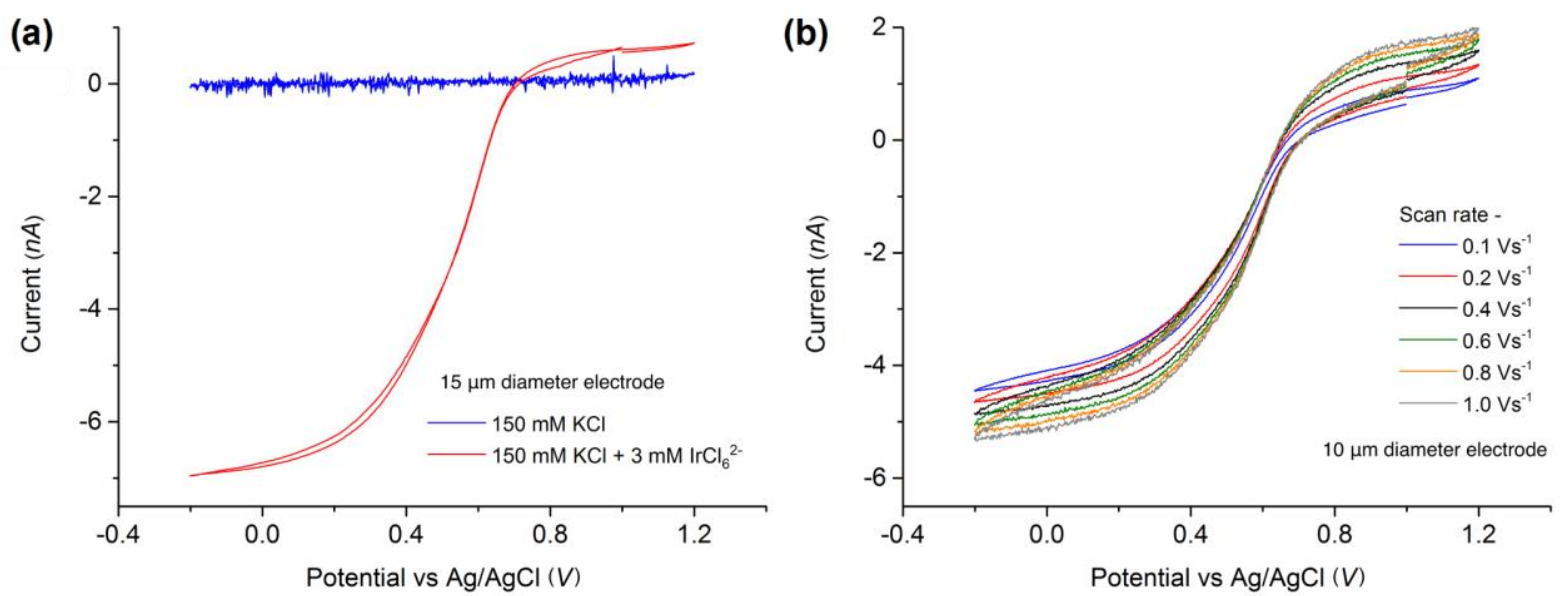

Figure $3-a) C V$ scans comparing the electrode response of supporting $150 \mathrm{mM} \mathrm{KCl}$ electrolyte only (blue) to supporting electrolyte with redox mediator (red). Scan rate - $10 \mathrm{mVs}^{-1}$, electrode diameter $15 \mu \mathrm{m}$. b) Repeat $\mathrm{CV}$ scans of $3 \mathrm{mM} \mathrm{IrCl}{ }_{6}^{2-}$ reduction in $150 \mathrm{mM} \mathrm{KCl}$ supporting electrolyte at increasing scan rates. Electrode diameter $-10 \mu \mathrm{m}$. For all CV scans, the potential was held at $1 \mathrm{~V}$ for 5 seconds before starting the scan and all data sets were filtered with FFT at $500 \mathrm{~Hz}$ for noise reduction.

, typically displaying fast electron transfer kinetics $\left(k_{0}>10 \times 10^{-3} \mathrm{cms}^{-1}\right)$ [34]. The cyclic voltammetry (CV) scan results are shown in Figure 3. The results demonstrate the expected behaviour of an UME, with no peaks in the response that are seen with macro-electrodes. The hemispherical diffusion of a reactant towards an UME leads to the characteristic steady state limiting current, where the reaction rate is constant and limited by the diffusion of fresh reactant towards the electrode surface. Repeated measurements with increasing $\mathrm{CV}$ scan rates show little change from $0.1-1 \mathrm{Vs}^{-1}$, apart from the expected increase in capacitive charging currents (indicated by an increasing area within the CV trace at larger scan rates). This steady state response is due to the increased reactant diffusion to the electrode surface in the hemispherical regime, requiring much higher scan rates to deplete the reactant within the diffusion layer, which would lead to the peaks in the response seen with the linear diffusion towards macro-electrodes. The reduction of $\mathrm{IrCl}_{6}{ }^{2-}$ is clearly distinguishable from the flat background recorded in blank $\mathrm{KCl}$ electrolyte, indicating that the recorded signal is due to redox current, with good agreement to the theoretical response.

The empirical formula for heterogeneous electron transfer at a flat, circular UME following ButlerVolmer kinetics [35], proposed by Oldham and Zoski [36], hereinafter referred to as the Oldham-Zoski formula, was fitted to the experimental data for the reduction of $\mathrm{IrCl}_{6}{ }^{2-}$. The Oldham-Zoski formula can be expressed as,

$i=\left(\frac{i_{d}}{\theta}\right) \cdot\left[1+\frac{\pi}{\kappa \theta}\left(\frac{2 \kappa \theta+3 \pi}{4 \kappa \theta+3 \pi^{2}}\right)\right]^{-1}$ 
Where $\kappa$ and $\theta$ are functions of the electrode potential, $E$,

$$
\begin{aligned}
& \kappa=\frac{\pi k^{0} r_{0}}{4 D_{o}} \exp \left[\frac{-\alpha n F}{R T}\left(E-E^{0^{\prime}}\right)\right] \\
& \theta=1+\exp \left[\frac{n F}{R T}\left(E-E^{0^{\prime}}\right)\right]
\end{aligned}
$$

Where $i$ is the electrode current, $i_{d}$ is the steady state current, $r_{o}$ is the electrode radius, $k_{0}$ is the standard transfer constant, $\alpha$ is the transfer coefficient, $D_{o}$ is the diffusion constant and $E^{0^{\prime}}$ is the formal potential for the redox reaction. A small correction in $i_{d}$ for the electrode recess into the substrate is included [36] (see Supporting Information S3).

It is often assumed when analysing electrochemical voltammograms that the transfer coefficient, $\alpha$, is an energy independent term and a constant equal to 0.5 , which is generally accurate when measurements are taken over small potential ranges on metallic surfaces, as any potential dependent effects are negligible [35]. However, the potential ranges measured in this study (> $700 \mathrm{meV}$ ) were larger than typically required to reach the steady state current seen in UME voltammograms [34]. When this was considered alongside the unique electronic properties of graphene, in particular the linear dispersion relation and small carrier concentration at the Dirac point, $\alpha$ was allowed to vary as a fitting parameter as the assumption that $\alpha=0.5$ proved incorrect for the system under study (see Supporting Information Figure 7). The value of $k_{0}$ was the main parameter of interest and was allowed to vary as a fitting parameter. The electrode radius, $r_{o}$, was also set as a variable fitting parameter as a way of accessing the effective area of the electrode undergoing electron transfer with the redox mediator. The diffusion constants for both the reduced and oxidised forms of the redox mediator were taken as $7.5 \times 10^{-6} \mathrm{~cm}^{2} \mathrm{~s}^{-1}$ [34]. The formal potential of the reaction, $E^{0^{\prime}}$, is unknown and needed to be independently estimated. To do this, a CV scan was performed using a $3 \mathrm{~mm}$ diameter basal plane high ordered pyrolytic graphite (HOPG) electrode in the same measurement solution as for the graphene electrodes. An estimation of $E^{0^{\prime}}$ is possible from the average potential of the reduction and oxidation peaks assuming $k_{0}$ is not very small (quasi-reversible kinetics) and the diffusion constants for both oxidised and reduced species are similar [35] (see Supporting Information S3 for further details). An example of the Oldham-Zoski formula fit to the experimental data is shown in Figure 4.

The data recorded at a CV scan rate of $100 \mathrm{mVs}^{-1}$ was analysed. Although slower scan rates are usually preferable due to reduced distortion from capacitive charging currents, the small size of electrolyte droplet on the device surface meant evaporation could be an issue over the longer measurement time scales required at lower scan rates. Furthermore, as the Au electrode covers the entire area of the 100 $\mathrm{nm}$ thick $\mathrm{SiN}_{\mathrm{x}}$ membrane, a capacitance is formed between the Au and the electrolyte solution across this dielectric. This parasitic signal in parallel to the electrode double layer capacitance meant background subtraction was unreliable and therefore all data was fitted as measured. Due to not 
removing this parasitic capacitance, the forward and backward scans do not exactly trace over each other, however the fitting procedure effectively fits to the average of these two signals. CV data recorded using a blank, $\mathrm{h}-\mathrm{BN}$ electrode in $\mathrm{KCl}$ electrolyte shows a linear sloped offset, also seen in the redox scans, which is thought to be due to the diffusion of ions in the electrolyte (see supporting

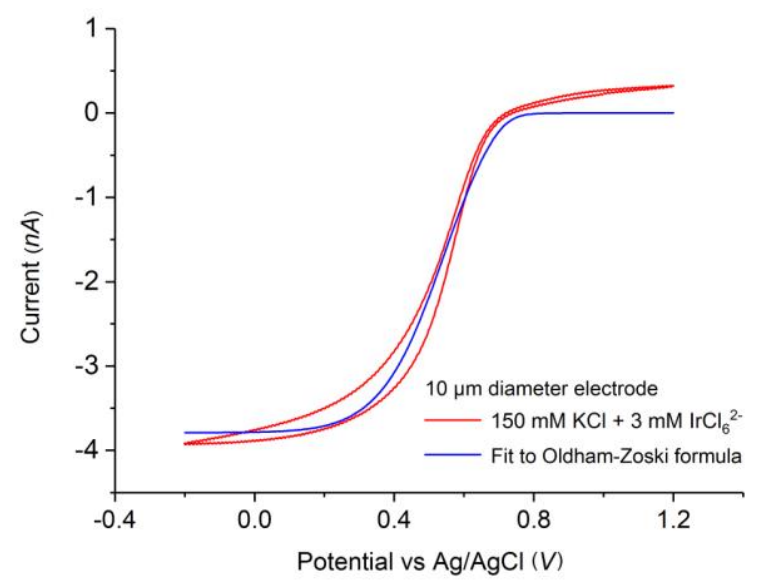

Figure $4-C V$ scan of $3 \mathrm{mM} \mathrm{IrCl}{ }_{6}^{2-}$ reduction in $150 \mathrm{mM} \mathrm{KCl}$ supporting electrolyte (blue) with Oldham-Zoski formula fit (red). Experimental data CV scan rate - $100 \mathrm{mVs}^{-1}$, electrode diameter $-10 \mu \mathrm{m}$. Fit parameters $-k^{0}=4.54 \times 10^{-}$ ${ }^{3} \mathrm{cms}^{-1}, \alpha=0.324, r_{o}=4.49 \mu \mathrm{m}$.

information S2). The scans have a slight anodic signal, presumed to be due to the presence of $\mathrm{IrCl}_{6}{ }^{3-}$ contamination in the solution, which can be expected due to spontaneous redox reactions of the redox couple, especially in the presence of light [37]. No corrections were made for uncompensated resistances as the current never exceeded $10 \mathrm{nA}$. It was assumed the Au-graphene contact resistance was the largest source of resistance to current flow (usually $<100 \mathrm{k} \Omega$ ) [38] and therefore voltage drops would be of the order of a few $\mathrm{mV}$ and therefore below the overall accuracy of the data.

Raman spectroscopy measurements were recorded before and after electrochemical measurement of the devices, shown in Figure 5(a-b). The graphene Raman spectrum is characterised by the G, D and 2D peaks [39]. The large intensity of the 2D peak observed compared to the G peak is typical of high quality, doping-free, monolayer graphene [40]. The $\mathrm{D}$ peak of graphene is indicative of defects and $\mathrm{sp}^{3}$ type bonds and is expected to occur around $1350 \mathrm{~cm}^{-1}$, which overlaps partially with the h-BN peak at $1365 \mathrm{~cm}^{-1}$; however, a perfectly symmetric h-BN peak and lack of a shoulder indicates that no graphene D peak is present, as seen in the inset. This allowed the initial quality of the graphene to be assessed once fabricated into a device structure, and then a comparison could be made once the device had been exposed to the electrolyte and undergone electrochemical electron transfer. All Raman spectra were similar between devices and no significant differences were seen between different areas of the electrode. Therefore, the spectra shown in Figure 5(a-b) were acquired in the approximate electrode centre position and can be regarded as representative for the entire active area. This is confirmed in Figure 4(c) which plots the 2D vs G positions for Raman spectra extracted from spatial maps before (red) and after (blue) the electrochemical measurements. We follow the analysis introduced in Refs. [41] and [42], which separates strain (along the black charge-neutral line) from doping (along the green strain-neutral line assuming p-doping) relative to the unstrained and undoped Raman frequencies 
(denoted by the star in Figure 5(c) [42, 43]). We find a similar behaviour for spectra taken before and after the electrochemical measurements: the data points scatter tightly around the black chargeneutrality line. This indicates tensile strain up to $0.25 \%$ together with minimal doping which varies

(a)

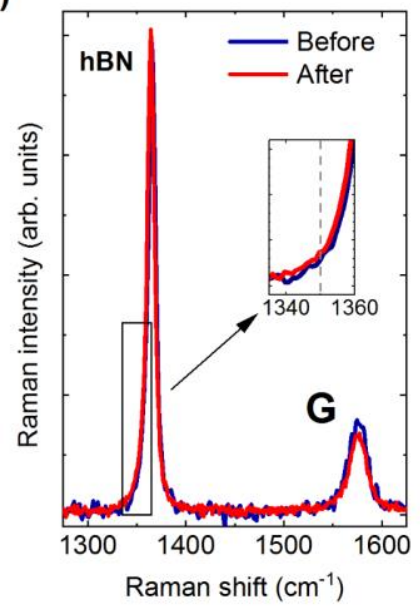

(b)

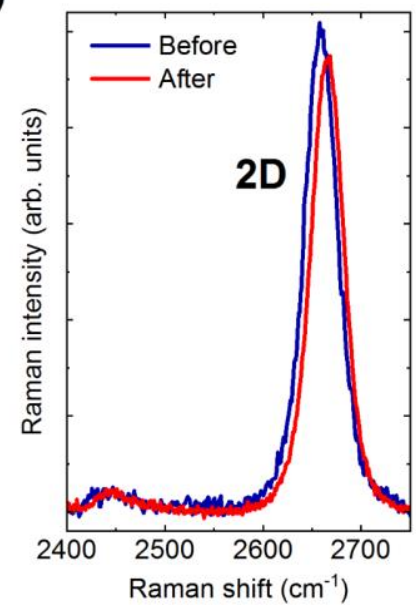

(c)

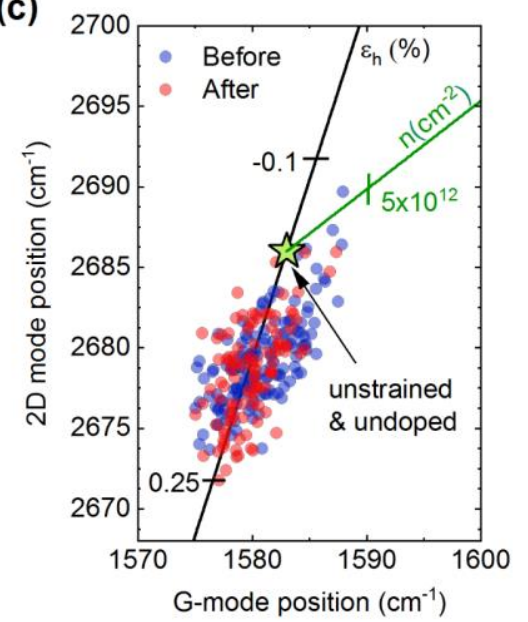

Figure 5 - A comparison of Raman spectra for a device before (blue) and after (red) electrochemical measurements focussing (a) on the spectral regions of the graphene $D \& G$-mode and the hBN Raman peak and (b) on the 2D-mode. Inset in (a) shows expected position of the D peak (grey dashed line). The laser wavelength used in $(a, b)$ was $532 \mathrm{~nm}$ and spectra were taken at the centre of the electrode. Raman shift resolution is $\sim 4 \mathrm{~cm}^{-1}$. FWHM(2D): Before - $36 \mathrm{~cm}^{-1}$, After-36 $\mathrm{cm}^{-1}$. FWHM(G): Before $-20 \mathrm{~cm}^{-1}$, After $-21 \mathrm{~cm}^{-1} . \operatorname{Pos}(2 D)$ : Before $-2660 \mathrm{~cm}^{-1}$, After $-2665 \mathrm{~cm}^{-1}$. Pos $(G):$ Before $-1575 \mathrm{~cm}^{-}$ ${ }^{1}$, After-1575 $\mathrm{cm}^{-1} . I(G) / I(2 D):$ Before - 0.1. I(G)/I(2D): After-0.1 Pos $(h-B N)-1365 \mathrm{~cm}^{-1}$. Pos $(D)-$ $1350 \mathrm{~cm}^{-1}$. (c) Correlation of $G$ - and 2D-mode positions extracted from a Raman map of the device to separate mechanical strain and charge doping show no major changes before (blue) and after (red) electrochemical measurements. For both cases, the strain varies between $0.25 \%$ and $-0.1 \%$ and doping varies between 0 and $5 \times 10^{12} \mathrm{~cm}^{-2}$ (assuming p-doping). The laser wavelength used in (c) was $514.5 \mathrm{~nm}$.

from 0 to $5 \times 10^{12} \mathrm{~cm}^{-2}$. Overall, the Raman data suggests a homogeneous electronic structure with little variation in strain and doping considering the overall size of the device.

\section{Discussion}

The electrochemical and Raman spectroscopy measurements presented here serve as confirmations that a pristine graphene UME was produced, free of polymer contamination and substrate charge effects, resulting very low doping, as well as with a well define area due to the self-aligned fabrication process. Nonetheless, these are preliminary measurements and there exist a number of second order effects that should be noted, resulting in discrepancies between measured and theoretical values. We discuss these effects and results here and note that having eliminated the large deviations arising from polymer/substrate doping and poorly defined electrode area, future experiments can isolate and control or eliminate these other effects. 
The measured current magnitude was slightly lower than expected on most devices, with the largest difference between recorded and theoretical values being $-10 \%$. The reason for this difference is thought to be due to the concentration of the redox mediator being lower than the value actually used, due to some $\mathrm{IrCl}_{6}{ }^{2-}$ spontaneously converting to $\mathrm{IrCl}_{6}^{3-}$ in the prepared solution [37]. The evidence for this lies in the fact the size of the anodic current is similar to the difference between the theoretical and measured cathodic current (see supporting information). Alongside this, a reduction in current could be due to areas of organic contamination adsorbed on graphene surfaces when exposed to the atmosphere, which is generally regarded as unavoidable and invariably seen in TEM images on the micron scale [20], however the effect of minor contamination should be small for outer sphere redox processes [44]. It is also possible that the previously reported values for the diffusion constant were not applicable to this setup and could have caused a systematic error in the current magnitude. Due to the small size of the electrode, and the fact that it needed to be removed from clean room conditions for measurement, means that other sources of particulate contamination cannot be completely ruled out. This lower than expected current magnitude is not expected to have a big effect on the electrochemical parameters deduced from the data [45].

The average values of the kinetic parameters $k_{0}$ and $\alpha$ for the reduction of $\mathrm{IrCl}_{6}{ }^{2-}$ are calculated as $3.04 \pm$ $0.78 \times 10^{-3} \mathrm{cms}^{-1}$ and $0.272 \pm 0.024$ respectively, taken from the Oldham-Zoski formula fit and averaged between 4 devices (full data available in Supporting Information Tables 1 and 2). The standard deviation in the range of results are $25 \%$ and $8.8 \%$ of the mean value for $k_{0}$ and $\alpha$ respectively. A relatively large spread in results is often seen for graphene devices, in particularly when air exposed [46] and this spread in $k_{0}$ could be reflecting the intrinsic sensitivity of pristine graphene to external factors such as atmospheric conditions and trace contaminants. The precise effect of atmospheric contaminants on graphene is still a subject of debate and is likely to play a role [46], but quantitative analysis is lacking. This effect will be present on any surface-exposed graphene sensor and therefore may limit the possible consistency between devices using pristine graphene. In this regard, highly doped or functionalised graphene samples could yield more consistency as small changes in charge carrier concentration due to contamination will have less of an effect on the overall DoS and hence electron transfer parameters.

When comparing the values of $k_{0}$ to similar previous data studying $\mathrm{IrCl}_{6}^{2-}$ redox with graphene electrodes, differences of around an order of magnitude are seen. The closest measurements made to those presented in this paper are the work of Velicky et al. [46]. In this study mechanically exfoliated flakes were deposited on $\mathrm{SiO}_{2}$ surfaces and the standard rate constant measured for $\mathrm{IrCl}_{6}{ }^{2-}$ redox on several monolayer graphene samples. Measurements were taken on the basal plane with no edge effect. The technique used means no extensive fabrication was required, as the graphene was contacted using Ag paint and the electrochemistry was performed in a small, deposited droplet, meaning that the surface was free from fabrication polymer contamination (although still exposed to atmospheric contamination). An average $k_{0}$ value of $34.8 \pm 0.47 \times 10^{-3} \mathrm{cms}^{-1}$ was measured, which is over an order 
of magnitude larger than the value measured for the samples presented in this paper. Another study [47] using a similar method but with the graphene on a polymer substrate measures $k_{0}$ as $3.08 \times 10^{-3} \mathrm{cms}^{-1}$, which is a very similar value to that measured on the samples presented in this paper. This method is suitable for laboratory studies of the properties of pristine graphene and confirms our results, but is not a fabrication route that yields realistic pristine graphene UMEs or devices with pre-defined electrode areas. We have summarised the values of $k_{0}$ and $\alpha$ obtained in this paper and others in literature in Table 1, and compared them to values for HOPG [44] and Pt [48] from literature.

Table 1: Comparative values for $k_{0}$ and $\alpha$ for graphene, $H O P G$ and $P t$

\begin{tabular}{|l|l|l|l|}
\hline Electrode (substrate) & $\boldsymbol{k}_{\boldsymbol{o}}\left(\mathbf{c m} \cdot \mathbf{s}^{-1}\right)$ & $\boldsymbol{\alpha}$ & $\boldsymbol{R e f}$ No. \\
\hline Exfoliated graphene $(\mathrm{h}-\mathrm{BN})$ & $3.04 \times 10^{-3}$ & 0.272 & This work \\
\hline Exfoliated graphene $\left(\mathrm{SiO}_{2}\right)$ & $34.8 \times 10^{-3}$ & 0.5 (assumed) & 46 \\
\hline Exfoliated graphene (polymer) & $3.08 \times 10^{-3}$ & 0.5 (assumed) & 47 \\
\hline HOPG & $>1.9$ & 0.5 (assumed) & 44 \\
\hline Platinum & 1.9 & 0.56 & 48 \\
\hline
\end{tabular}

It can be suggested that the substrate may play a key role in the difference between the values for graphene on $\mathrm{h}-\mathrm{BN}$ or polymer substrates and $\mathrm{SiO}_{2}$ substrates. The effect of increased chemical reactivity on certain substrate surfaces is well established [24], with charged impurities causing substrate doping effects that cause the higher reactivity. Uncharged polymers and h-BN both have low charge defect density where as $\mathrm{SiO}_{2}$ has a high charge defect density. The effect of charge density on electrostatic reactivity has been further confirmed by controlled electrostatic doping [49]. The lower than expected $k_{0}$ values for graphene on h-BN (and uncharged polymers) is thought to be due to this reduced charged defect doping. In particular, reducing contamination from polymers used during fabrication would usually lead to an increased $k_{0}$ as contamination acts as a potential barrier to electron transfer and hence slower kinetics would be expected. These results show the opposite trend. Furthermore, the fact that h$\mathrm{BN}$ is atomically flat is thought to only play a small role, as high reactivity has been seen on atomically smooth mica [23], which has a charged surface and therefore suggesting that charged impurities are a bigger factor than surface roughness.

The assumption in many studies that the transfer coefficient, $\alpha$, is an energy independent term equalling 0.5 is also seen to be in doubt on pristine, un-doped graphene, as all the values recorded in this study were consistently lower than this. Over the large potential ranges needed to reach steady state current for these measurements $(>700 \mathrm{meV})$, it is likely that $\alpha$ would not be a constant and should be represented as a potential dependent parameter, something that is not taken into account in the Butler-Volmer theory [35]. This should to be taken into account for future work on graphene, especially when slow kinetic processes are being measured over large potential ranges on samples with low doping [45]. Further study would require the application of a more robust theoretical model, such as Marcus-Gerischer theory, taking into account graphene's negligible DoS at the Dirac point and linear change in electronic 
DoS with potential at low energies, which is thought to be the most likely cause of $\alpha$ appearing to be a function of potential in these results. However, to accurately apply Marcus-Gerischer theory, the DoS as a function of electrode potential would need to be determined experimentally, requiring extensive measurements using a variety of redox couples, which was beyond the scope of these initial measurements.

The Raman spectroscopy shows the graphene was of high quality throughout. There is no detectable D peak and the $\mathrm{I}(\mathrm{G}) / \mathrm{I}(2 \mathrm{D})$ ratio of 0.1 is consistent with highly pristine monolayer graphene, with a ratio of less than 0.3 being considered low doping [40]. The statistical plot of G peak position vs 2D peak position also confirms the low doping levels that remain unchanged after electrochemical measurement. There is no D peak detectable before or after electrochemical measurement, ruling out the effect of defects on the electrochemical reduction measurements and implying that the electrolyte causes no significant damage (in the form of $s p^{3}$ defects) to the graphene during these measurements. The effect of nanoscale defects cannot be ruled out as the spatial resolution of Raman is $\sim 0.5 \mu \mathrm{m}$. However, a degree of nanoscale defectiveness is always observable in graphene and is unavoidable [31].

The Raman spectra all show some broadening and downshifting of the $G$ and 2D peaks beyond the typically measured values $[39,50]$. Statistical analysis of the $G$ and $2 D$ peak positions shows that these values are not consistent with significant doping changes and are primarily caused by strain in the graphene, which can cause peak broadening and downshifting [51]. The strain is believed to originate from transferring the h-BN-graphene layer over the hole in the $\mathrm{SiN}_{\mathrm{x}}$ substrate, with vdW forces pulling the suspended structure inwards towards the substrate. This could be measured with AFM, with the flakes deforming around $50 \mathrm{~nm}$ into a $10 \mu \mathrm{m}$ diameter hole when the h-BN was $70 \mathrm{~nm}$ thick. This strain is not uniaxial or constant over the Raman excitation area and therefore represents a complex picture for analysis. An estimation of the hydrostatic strain components from the peak positions yields a value in the range of $0-0.25 \%$, which is not known to have any significant effect on the electronic structure of graphene [50] and is consistent with the AFM values recorded. The slight upshift in 2D peak position after electrochemical measurements could be due to a small rearrangement of this strain after contact with the electrolyte, although this upshift value is on the very limit of the spectral resolution of the Raman system and within the range of sample-to-sample and point-to-point variations.

As the fabrication method presents a clean, pristine surface, the graphene could be accurately functionalised either before or after being incorporated into the device structure, offering flexibility that is not possible with devices requiring polymer-based lithography. Functionalisation with a variety of biomolecules is necessary to tailor an electrochemical sensor for high specificity [52]. The device could also be structured to include heterostructures of different two-dimensional (2-D) materials, such as the transition metal dichalcogenides, allowing the investigation of fundamental electrochemistry and potential applications. This device structure could be scaled into multiple arrays using a variety of 
microsystems fabrication techniques. Arrays could be individually functionalised, creating multiple sensing elements on a single chip, alongside the possibility of delivering analytes via microfluidics. Moreover, this fabrication technique can be scaled up with $100 \%$ yield across large arrays of suspended devices to the stability provided by the dielectric and polymer support layer as has been demonstrated for other suspended graphene microsystem devices [53,54].

\section{Conclusion}

A fabrication method for making electrical contact and defining the electrochemically active area in graphene without exposure to lithography or transfer polymers is demonstrated here, and validated by Raman spectroscopy and measuring the correct current densities for the reduction of $\mathrm{IrCl}_{6}{ }^{2-}$. The expected characteristics of a UME were observed with increasing CV scan rate having little effect on the electrochemical response and the theoretical Oldham-Zoski formula showing a good fit to the experimental data. The Raman spectra showed the graphene to be of high crystalline quality and low doping, both before and after electrochemical measurements, showing that the fabrication methods and redox measurements induced negligible defectiveness or doping into the graphene.

Comparisons to results in literature suggests the h-BN substrate chosen here reduces the standard rate constant, $k_{0}$, due to reduction in substrate doping. Further independent study on well-defined macroelectrodes should confirm this effect, as this would allow alternate analysis methods based on peak positions to be applied. The values of the standard rate constant showed relatively large variation between devices, likely a result graphene's susceptibility to varying environmental conditions and due to variations in the potential of the Dirac point across different flakes. The results also highlighted that values of the transfer coefficient, $\alpha$, were consistently less than the commonly assumed value of 0.5 and likely to be a function of electrode potential.

Removing the parasitic capacitance through the $\mathrm{SiN}_{\mathrm{x}}$ substrate would allow new measurements including impedance measurements to probe the sensing capabilities of the novel electrode structure to be carried out. It is expected this is where the true sensitivity of this device could be utilised, especially if modified into a field effect transistor (FET) type device and observing the quantum capacitance changes due to analyte interactions [55]. This could then be interfaced with microfluidics to allow a continuous supply of various analytes to a single (-or array of) devices. This device structure would also be compatible with surface functionalisation or organic solvents to allow more specific or novel sensing measurements to be performed.

\section{Acknowledgements}

The authors acknowledge funding from the Engineering \& Physical Sciences Research Council (EPSRC) grant EP/G03737X/1. The authors acknowledge Matej Velický for helpful discussions. 


\section{Author Contributions}

All authors conceived and designed the experiments. S.G. performed the device fabrication, characterisation and analysis, together with Z.C. for the electrochemistry and S.H. for the Raman spectroscopy. The manuscript was written by S.G. and A.V., with contributions from B.G., E.H. and S.H., and reviewed and approved by all authors.

\section{References}

[1] K.S. Novoselov, A.K. Geim, S.V. Morozov, D. Jiang, Y. Zhang, S.V. Dubonos, I.V. Grigorieva, A.A. Firsov, Electric Field Effect in Atomically Thin Carbon Films, Science 306(5696) (2004) 666. [2] E.W. Hill, A. Vijayaragahvan, K. Novoselov, Graphene Sensors, IEEE Sensors Journal 11(12) (2011) 3161-3170.

[3] S.K. Vashist, J.H.T. Luong, Recent advances in electrochemical biosensing schemes using graphene and graphene-based nanocomposites, Carbon 84 (2015) 519-550.

[4] A.M. Bond, K.B. Oldham, C.G. Zoski, Steady-state voltammetry, Analytica Chimica Acta 216 (1989) 177-230.

[5] H. Pajouhi, A.Y. Jou, R. Jain, A. Ziabari, A. Shakouri, C.A. Savran, S. Mohammadi, Flexible complementary metal oxide semiconductor microelectrode arrays with applications in single cell characterization, Applied Physics Letters 107(20) (2015) 203103.

[6] K. Jedlińska, R. Porada, J. Lipińska, B. Baś, The bi-band silver microelectrode: Fabrication, characterization and analytical study, Sensors and Actuators B: Chemical 302 (2020) 127152.

[7] K.S. Novoselov, V.I. Fal'ko, L. Colombo, P.R. Gellert, M.G. Schwab, K. Kim, A roadmap for graphene, Nature 490(7419) (2012) 192-200.

[8] Z. Cheng, Q. Zhou, C. Wang, Q. Li, C. Wang, Y. Fang, Toward Intrinsic Graphene Surfaces: A Systematic Study on Thermal Annealing and Wet-Chemical Treatment of SiO2-Supported Graphene Devices, Nano Letters 11(2) (2011) 767-771.

[9] A. Bonanni, M. Pumera, Surfactants used for dispersion of graphenes exhibit strong influence on electrochemical impedance spectroscopic response, Electrochemistry Communications 16(1) (2012) 19-21.

[10] Y.-C. Lin, C.-C. Lu, C.-H. Yeh, C. Jin, K. Suenaga, P.-W. Chiu, Graphene Annealing: How Clean Can It Be?, Nano Letters 12(1) (2012) 414-419.

[11] H.V. Patten, M. Velický, N. Clark, C.A. Muryn, I.A. Kinloch, R.A.W. Dryfe, Electrochemistry of well-defined graphene samples: role of contaminants, Faraday Discussions 172(0) (2014) 261-272. [12] D.C. Elias, R.R. Nair, T.M.G. Mohiuddin, S.V. Morozov, P. Blake, M.P. Halsall, A.C. Ferrari, D.W. Boukhvalov, M.I. Katsnelson, A.K. Geim, K.S. Novoselov, Control of Graphene\&\#039;s Properties by Reversible Hydrogenation: Evidence for Graphane, Science 323(5914) (2009) 610. [13] S.J. Park, O.S. Kwon, S.H. Lee, H.S. Song, T.H. Park, J. Jang, Ultrasensitive Flexible Graphene Based Field-Effect Transistor (FET)-Type Bioelectronic Nose, Nano Letters 12(10) (2012) 50825090.

[14] S.P. Koenig, L. Wang, J. Pellegrino, J.S. Bunch, Selective molecular sieving through porous graphene, Nature Nanotechnology 7(11) (2012) 728-732.

[15] K.J. Ziegler, Z. Gu, H. Peng, E.L. Flor, R.H. Hauge, R.E. Smalley, Controlled Oxidative Cutting of Single-Walled Carbon Nanotubes, Journal of the American Chemical Society 127(5) (2005) 15411547.

[16] Y. Yu Wang, P.J. Burke, A large-area and contamination-free graphene transistor for liquidgated sensing applications, Applied Physics Letters 103(5) (2013) 052103.

[17] G. Zhang, A.G. Güell, P.M. Kirkman, R.A. Lazenby, T.S. Miller, P.R. Unwin, Versatile Polymer-Free Graphene Transfer Method and Applications, ACS Applied Materials \& Interfaces 8(12) (2016) 8008-8016. 
[18] W.-H. Lin, T.-H. Chen, J.-K. Chang, J.-I. Taur, Y.-Y. Lo, W.-L. Lee, C.-S. Chang, W.-B. Su, C.I. Wu, A Direct and Polymer-Free Method for Transferring Graphene Grown by Chemical Vapor Deposition to Any Substrate, ACS Nano 8(2) (2014) 1784-1791.

[19] A.V. Kretinin, Y. Cao, J.S. Tu, G.L. Yu, R. Jalil, K.S. Novoselov, S.J. Haigh, A. Gholinia, A. Mishchenko, M. Lozada, T. Georgiou, C.R. Woods, F. Withers, P. Blake, G. Eda, A. Wirsig, C. Hucho, K. Watanabe, T. Taniguchi, A.K. Geim, R.V. Gorbachev, Electronic Properties of Graphene Encapsulated with Different Two-Dimensional Atomic Crystals, Nano Letters 14(6) (2014) 32703276.

[20] S.J. Haigh, A. Gholinia, R. Jalil, S. Romani, L. Britnell, D.C. Elias, K.S. Novoselov, L.A. Ponomarenko, A.K. Geim, R. Gorbachev, Cross-sectional imaging of individual layers and buried interfaces of graphene-based heterostructures and superlattices, Nature Materials 11(9) (2012) 764767.

[21] Y. Zhang, V.W. Brar, C. Girit, A. Zettl, M.F. Crommie, Origin of spatial charge inhomogeneity in graphene, Nature Physics 5(10) (2009) 722-726.

[22] J. Martin, N. Akerman, G. Ulbricht, T. Lohmann, J.H. Smet, K. von Klitzing, A. Yacoby, Observation of electron-hole puddles in graphene using a scanning single-electron transistor, Nature Physics 4(2) (2008) 144-148.

[23] M. Yamamoto, T.L. Einstein, M.S. Fuhrer, W.G. Cullen, Charge Inhomogeneity Determines Oxidative Reactivity of Graphene on Substrates, ACS Nano 6(9) (2012) 8335-8341.

[24] Q.H. Wang, Z. Jin, K.K. Kim, A.J. Hilmer, G.L.C. Paulus, C.-J. Shih, M.-H. Ham, J.D. SanchezYamagishi, K. Watanabe, T. Taniguchi, J. Kong, P. Jarillo-Herrero, M.S. Strano, Understanding and controlling the substrate effect on graphene electron-transfer chemistry via reactivity imprint lithography, Nature Chemistry 4(9) (2012) 724-732.

[25] M. Lafkioti, B. Krauss, T. Lohmann, U. Zschieschang, H. Klauk, K.v. Klitzing, J.H. Smet, Graphene on a Hydrophobic Substrate: Doping Reduction and Hysteresis Suppression under Ambient Conditions, Nano Letters 10(4) (2010) 1149-1153.

[26] A.T. Valota, I.A. Kinloch, K.S. Novoselov, C. Casiraghi, A. Eckmann, E.W. Hill, R.A.W. Dryfe, Electrochemical Behavior of Monolayer and Bilayer Graphene, ACS Nano 5(11) (2011) 8809-8815.

[27] C.R. Dean, A.F. Young, I. Meric, C. Lee, L. Wang, S. Sorgenfrei, K. Watanabe, T. Taniguchi, P. Kim, K.L. Shepard, J. Hone, Boron nitride substrates for high-quality graphene electronics, Nature Nanotechnology 5(10) (2010) 722-726.

[28] P. Wei, J. Shen, K. Wu, N. Yang, Defect-dependent electrochemistry of exfoliated graphene layers, Carbon 154 (2019) 125-131.

[29] B. Körbitzer, P. Krauß, S. Belle, J.J. Schneider, C. Thielemann, Electrochemical Characterization of Graphene Microelectrodes for Biological Applications, ChemNanoMat 5(4) (2019) 427-435.

[30] F. Schedin, A.K. Geim, S.V. Morozov, E.W. Hill, P. Blake, M.I. Katsnelson, K.S. Novoselov, Detection of individual gas molecules adsorbed on graphene, Nature Materials 6(9) (2007) 652-655. [31] S. Hu, M. Lozada-Hidalgo, F.C. Wang, A. Mishchenko, F. Schedin, R.R. Nair, E.W. Hill, D.W. Boukhvalov, M.I. Katsnelson, R.A.W. Dryfe, I.V. Grigorieva, H.A. Wu, A.K. Geim, Proton transport through one-atom-thick crystals, Nature 516(7530) (2014) 227-230.

[32] L. Wang, I. Meric, P.Y. Huang, Q. Gao, Y. Gao, H. Tran, T. Taniguchi, K. Watanabe, L.M. Campos, D.A. Muller, J. Guo, P. Kim, J. Hone, K.L. Shepard, C.R. Dean, One-Dimensional Electrical Contact to a Two-Dimensional Material, Science 342(6158) (2013) 614.

[33] E.J. Dickinson, J.G. Limon-Petersen, N.V. Rees, R.G. Compton, How much supporting electrolyte is required to make a cyclic voltammetry experiment quantitatively "diffusional"? A theoretical and experimental investigation, The Journal of Physical Chemistry C 113(25) (2009) 11157-11171.

[34] C. Beriet, D. Pletcher, A further microelectrode study of the influence of electrolyte concentration on the kinetics of redox couples, Journal of Electroanalytical Chemistry 375(1) (1994) 213-218.

[35] M.R. de Rooij Dr, Electrochemical Methods: Fundamentals and Applications, Anti-Corrosion Methods and Materials 50(5) (2003).

[36] K.B. Oldham, C.G. Zoski, Comparison of voltammetric steady states at hemispherical and disc microelectrodes, Journal of Electroanalytical Chemistry and Interfacial Electrochemistry 256(1) (1988) 11-19. 
[37] S. Weusten, M. de Groot, J. van der Schaaf, A comparative study of the stability of hexachloroiridate and hexacyanoferrate in electrochemical mass transfer measurements, Journal of Electroanalytical Chemistry 878 (2020) 114512.

[38] F. Xia, V. Perebeinos, Y.-m. Lin, Y. Wu, P. Avouris, The origins and limits of metal-graphene junction resistance, Nature Nanotechnology 6(3) (2011) 179-184.

[39] A.C. Ferrari, J.C. Meyer, V. Scardaci, C. Casiraghi, M. Lazzeri, F. Mauri, S. Piscanec, D. Jiang, K.S. Novoselov, S. Roth, A.K. Geim, Raman Spectrum of Graphene and Graphene Layers, Physical Review Letters 97(18) (2006) 187401.

[40] C. Casiraghi, S. Pisana, K.S. Novoselov, A.K. Geim, A.C. Ferrari, Raman fingerprint of charged impurities in graphene, Applied Physics Letters 91(23) (2007) 233108.

[41] J.E. Lee, G. Ahn, J. Shim, Y.S. Lee, S. Ryu, Optical separation of mechanical strain from charge doping in graphene, Nature communications 3(1) (2012) 1-8.

[42] N.S. Mueller, S. Heeg, M.P. Alvarez, P. Kusch, S. Wasserroth, N. Clark, F. Schedin, J.

Parthenios, K. Papagelis, C. Galiotis, Evaluating arbitrary strain configurations and doping in graphene with Raman spectroscopy, 2D Materials 5(1) (2017) 015016.

[43] G. Froehlicher, S. Berciaud, Raman spectroscopy of electrochemically gated graphene transistors: Geometrical capacitance, electron-phonon, electron-electron, and electron-defect scattering, Physical Review B 91(20) (2015) 205413.

[44] G. Zhang, A.S. Cuharuc, A.G. Güell, P.R. Unwin, Electrochemistry at highly oriented pyrolytic graphite (HOPG): lower limit for the kinetics of outer-sphere redox processes and general implications for electron transfer models, Physical Chemistry Chemical Physics 17(17) (2015) 1182711838.

[45] Y. Liu, S. Chen, Electronic structure related electric-double-layer effects on heterogeneous ET kinetics on graphene electrode, Journal of Electroanalytical Chemistry 753 (2015) 3-8.

[46] M. Velický, D.F. Bradley, A.J. Cooper, E.W. Hill, I.A. Kinloch, A. Mishchenko, K.S. Novoselov, H.V. Patten, P.S. Toth, A.T. Valota, S.D. Worrall, R.A.W. Dryfe, Electron Transfer Kinetics on Mono- and Multilayer Graphene, ACS Nano 8(10) (2014) 10089-10100.

[47] P.S. Toth, A.T. Valota, M. Velický, I.A. Kinloch, K.S. Novoselov, E.W. Hill, R.A.W. Dryfe, Electrochemistry in a drop: a study of the electrochemical behaviour of mechanically exfoliated graphene on photoresist coated silicon substrate, Chemical Science 5(2) (2014) 582-589.

[48] J.V. Macpherson, C.E. Jones, P.R. Unwin, Radial flow microring electrode: investigation of fast heterogeneous electron-transfer processes, The Journal of Physical Chemistry B 102(49) (1998) 98919897.

[49] K.-W. Chang, I.A. Santos, Y. Nguyen, Y.-H. Su, C.C. Hsu, Y.-P. Hsieh, M. Hofmann, Electrostatic Control over the Electrochemical Reactivity of Graphene, Chemistry of Materials 30(20) (2018) 7178-7182.

[50] J. Zabel, R.R. Nair, A. Ott, T. Georgiou, A.K. Geim, K.S. Novoselov, C. Casiraghi, Raman Spectroscopy of Graphene and Bilayer under Biaxial Strain: Bubbles and Balloons, Nano Letters 12(2) (2012) 617-621.

[51] T.M.G. Mohiuddin, A. Lombardo, R.R. Nair, A. Bonetti, G. Savini, R. Jalil, N. Bonini, D.M. Basko, C. Galiotis, N. Marzari, K.S. Novoselov, A.K. Geim, A.C. Ferrari, Uniaxial strain in graphene by Raman spectroscopy: \$G\$ peak splitting, Grl"uneisen parameters, and sample orientation, Physical Review B 79(20) (2009) 205433.

[52] Y. Shao, J. Wang, H. Wu, J. Liu, I.A. Aksay, Y. Lin, Graphene based electrochemical sensors and biosensors: a review, Electroanalysis: An International Journal Devoted to Fundamental and Practical Aspects of Electroanalysis 22(10) (2010) 1027-1036.

[53] C. Berger, M. Dirschka, A. Vijayaraghavan, Ultra-thin graphene-polymer heterostructure membranes, Nanoscale 8(41) (2016) 17928-17939.

[54] C. Berger, R. Phillips, A. Centeno, A. Zurutuza, A. Vijayaraghavan, Capacitive pressure sensing with suspended graphene-polymer heterostructure membranes, Nanoscale 9(44) (2017) 17439-17449. [55] J. Xia, F. Chen, J. Li, N. Tao, Measurement of the quantum capacitance of graphene, Nature Nanotechnology 4(8) (2009) 505-509. 


\section{Supporting Information}

\section{Fabrication and electrochemical response of pristine graphene ultramicroelectrodes}

Stefan Goodwin ${ }^{1}$, Zachary Coldrick ${ }^{1}$, Sebastian Heeg ${ }^{2,3, \dagger}$, Bruce Grieve ${ }^{1}$, Aravind Vijayaraghavan ${ }^{2,3, *}$, Ernie W Hill ${ }^{3,4}$

${ }^{1}$ Department of Electrical and Electronic Engineering, The University of Manchester, Oxford Road, Manchester M13 9PL, UK

${ }^{2}$ Department of Materials, The University of Manchester, Oxford Road, Manchester M13 9PL, UK

${ }^{3}$ National Graphene Institute, The University of Manchester, Oxford Road, Manchester M13 9PL, UK

${ }^{4}$ Department of Computer Science, The University of Manchester, Oxford Road, Manchester, M13 9PL, UK.

$\dagger$ Present address: Department of Physics, Freie Universität Berlin, 14195 Berlin, Germany

* Corresponding author: aravind@manchester.ac.uk

\section{S1. Graphene device fabrication methods - further information}

\section{Dry etching of silicon nitride}

$\mathrm{SiN}_{\mathrm{x}}$ etching was carried out using reactive ion etching (RIE)[1], with optical resist (Shipley S1805) acting as a mask to protect chosen areas of the sample from the plasma. Using optical lithography, a pattern was created in $500 \mathrm{~nm}$ optical resist on one side of a clean, double side $\mathrm{SiN}_{\mathrm{x}}$ coated silicon wafer $\left(\mathrm{SiN}_{\mathrm{x}} / \mathrm{Si} / \mathrm{SiN}_{\mathrm{x}}\right)$, leaving the wafer surface with some areas protected by polymer resist and others exposed. The resist patterned sample was then placed into the vacuum chamber of the RIE apparatus, and exposed the sample to a $\mathrm{CHF}_{3} / \mathrm{SF}_{6}$ plasma for $\sim 2$ minutes removing the exposed $\mathrm{SiN}$ coating. The areas of the sample protected by resist were unaffected by this process, transferring the optically exposed lithography pattern into the $\mathrm{SiN}_{\mathrm{x}}$ coating of the silicon wafer. Immediately after the $\mathrm{CHF}_{3} / \mathrm{SF}_{6}$ plasma, the sample was exposed to an $\mathrm{O}_{2} /$ Ar plasma for 2 minutes, removing the polymer resist. This $\mathrm{O}_{2} /$ Ar plasma step does not extensively react with $\mathrm{Si}$ or $\mathrm{SiN}_{\mathrm{x}}$ and therefore does not affect the pattern made in the previous step.

\section{Wet etching of silicon}

Silicon was etched with $\mathrm{KOH}$ solutions giving an anisotropic etch, selective to the different crystal planes of monocrystalline silicon. The etch ratios for the $\langle 111: 110: 100\rangle$ crystal planes are approximately 1:600:400 [2]. If the wafer surface is terminated in the $\langle 100\rangle$ plane then the etching will proceed to create a pyramidal or trapezoid shaped trench, as shown in Figure S1. KOH has a negligible etch rate on $\mathrm{SiN}_{\mathrm{x}}$ and therefore a layer of $\mathrm{SiN}_{\mathrm{x}}$ serves as an etch-stop to protect parts of the silicon that 
do not require etching. If the silicon wafer has a layer of $\mathrm{SiN}_{\mathrm{x}}$ on both sides then etching the whole way through the silicon will leave a window or membrane of $\mathrm{SiN}_{\mathrm{x}}$ remaining on the opposite side to that where the etch started.

To perform the etch, aqueous $\mathrm{KOH}$ at a concentration of $30 \%$ w/v was heated to $80{ }^{\circ} \mathrm{C}$ with an external water bath and thermometer controlling the temperature. The silicon substrate with RIE patterned $\mathrm{SiN}_{\mathrm{x}}$ etch mask was placed in the solution, taking around 2 hours to etch through $300 \mu \mathrm{m}$ of silicon. The end point of the etching is determined by visual inspection, when the silicon has been entirely etched through the wafer, the remaining $\mathrm{SiN}_{\mathrm{x}}$ window becomes visible. Once complete the substrate was removed from

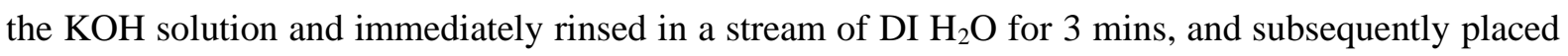
in a fresh beaker of $\mathrm{DI}_{2} \mathrm{O}$ for 12 hours to ensure no $\mathrm{KOH}$ deposits remained on the sample. An IPA rinse for 1 min followed by drying in a $\mathrm{N}_{2}$ gas flow finished the etching process.

1

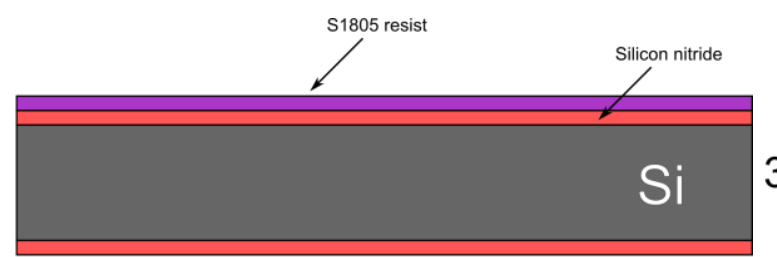

3

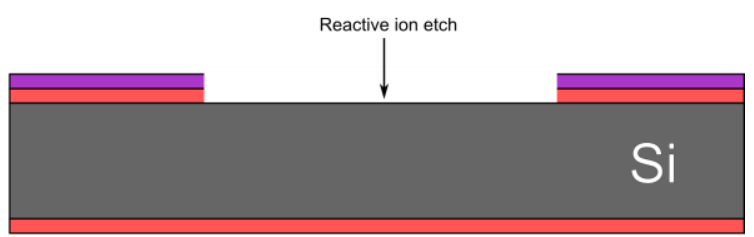

S1805 optical exposure and develop
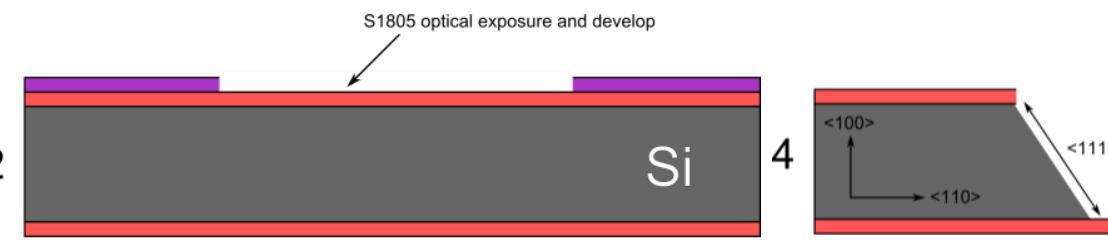

Wet KOH etch

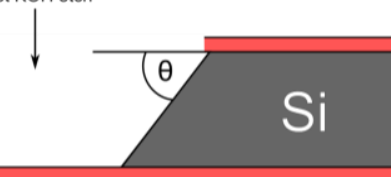

Figure S1 - Etching of silicon/silicon nitride substrates to create a silicon nitride membrane. $\theta=54.7^{\circ}$.

\section{HMDS monolayer deposition}

HMDS (hexamethyldisilazane) is used to create hydrophobic surfaces on silicon based materials. The HMDS molecule chemically reacts with hydroxyl $(\mathrm{OH})$ groups on surfaces, grafting a layer of methyl $\left(\mathrm{CH}_{3}\right)$ groups from the HMDS molecules to the surface. Methyl groups are non-polar and therefore give the surface hydrophobic properties. When $\mathrm{SiN}_{\mathrm{x}}$ surfaces are exposed to an oxygen plasma, the surface is functionalised with hydroxyl groups, which can in turn be exposed to vapour phase HMDS to allow the methyl grafting to occur.

For HMDS deposition, the $\mathrm{SiN}_{\mathrm{x}}$ surface initially required treatment in $\mathrm{O}_{2} / \mathrm{Ar}$ plasma, leaving the surface terminated with hydroxyl groups. The substrate was then rapidly transferred to a large petri dish containing $\sim 100 \mu \mathrm{L}$ of HMDS liquid in a small glass vial. The vial was placed on its side and was left unsealed, allowing the HMDS to evaporate and fill the petri dish. The lid of the petri dish containing both the substrate and HMDS filled vial was then sealed with plastic paraffin tape (Parafilm) to prevent the gaseous HMDS escaping. The petri dish was left for at least 4 hours at room temperature to allow 
the reaction to proceed. After this time the substrates were removed and were ready for further processing.

\section{Electrode fabrication}

Figures S2 shows example images of flake preparation and transfer steps. The dark field (DF) images are used to assess the initial quality of the prepared flakes, as large defects, edges and holes all show up brightly, with bubbles also appearing but with reduced intensity compared to edges. Using DF imaging, an absence of areas within the flake comparable in brightness to the edges means that area of a flake can be selected as the major-defect free region to act as the electrochemical electrode. These regions are indicated as red circles in Figure S2, demonstrating how the available area gets smaller after each processing step, due to thick h-BN flakes typically being smaller than graphene flakes, and the number of defects increasing with each transfer step.

\section{Chip carrier modification and bonding}

To make measurements with the electrochemical devices they needed to be connected to an external measurement system. For this purpose a modified chip carrier was used. Due to the double sided nature of this device, one side for contacts and the other side for electrochemical measurements, the chip carrier needed to be modified. For this purpose, a hole was drilled through the bottom of each chip carrier, with the hole being slightly smaller in diameter than the internal cavity width of the chip carrier itself, shown in Figure S4.

The device could then be mounted in the chip carrier, using a small droplet of PMMA ( $8 \%$ in anisole) at the edges for adhesion, making sure no PMMA came into contact with the graphene/electrode. The device was mounted so that the etched trench in the silicon aligned in the centre of the hole in the chip carrier, therefore the active graphene area could be accessed through the hole in the chip carrier. To make electrical contact between the chip carrier and the device, ultrasonic bonding was used to connect a fine wire between the small electrical contact on the device surface and the pads on the chip carrier. The chip carrier was then attached to a glass slide coated in aluminium with double sided adhesive tape, with the sensing surface facing upwards to allow access for measurements. This contacting method required no polymers or solvents and is therefore was the cleanest possible way to make an electrical contact.

\section{S2. Electrode characterisation - further information}

\section{Electrochemical measurement apparatus}

The devices were measured on a custom electrochemical measurement system. The system used a micromanipulator on which a holder for a $1.2 \mathrm{~mm}$ diameter borosilicate glass pipette could be attached, allowing the pipette to be positioned to an accuracy of $10 \mu \mathrm{m}$. The pipette end was shaped using a 


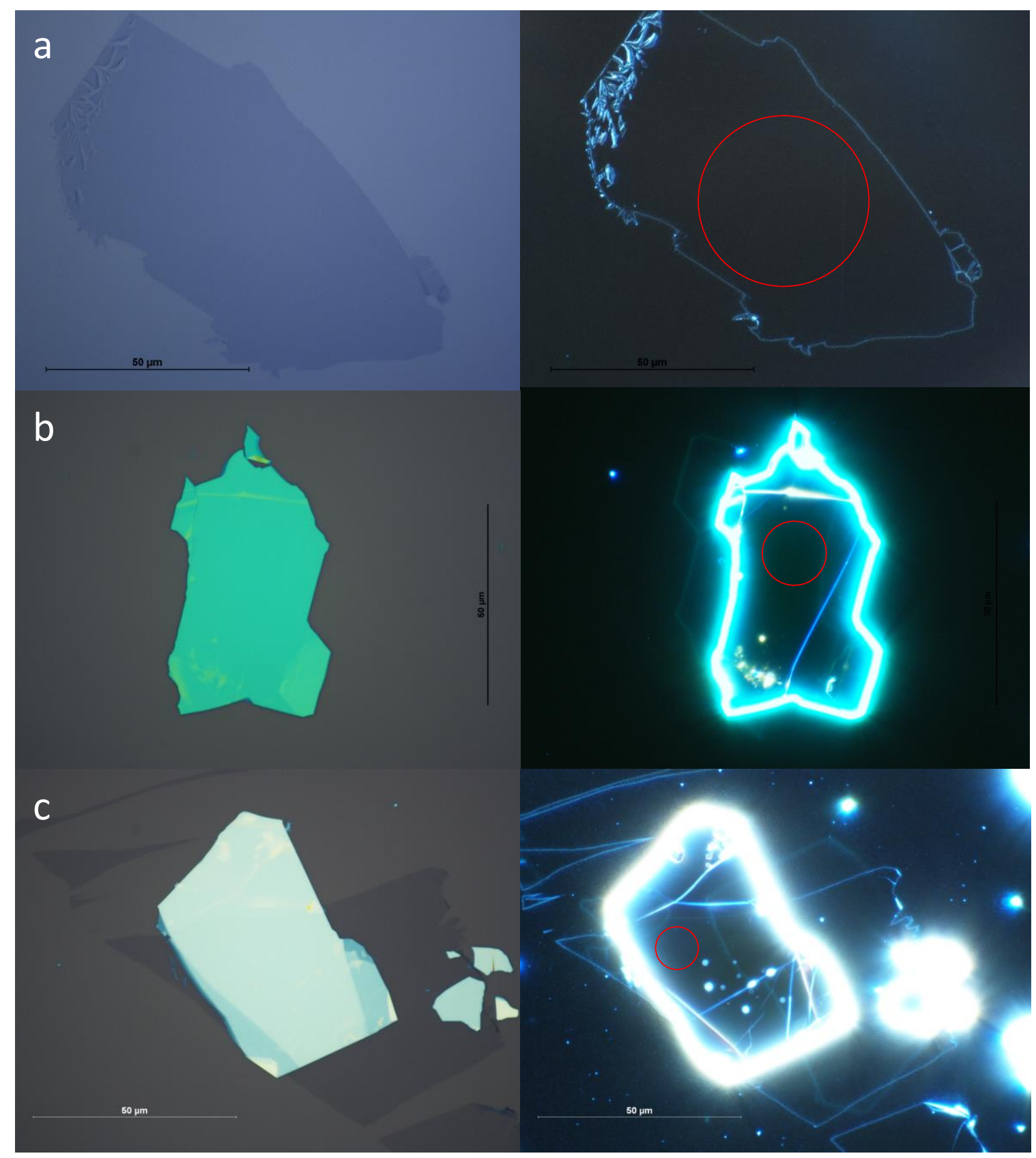

Figure S2 - Optical images of 2-D crystals. a-Mechanically exfoliated monolayer graphene flake on $90 \mathrm{~nm} \mathrm{SiO} 2$ (left) with equivalent DF image of a (right). $b$ - Mechanically exfoliated $h$-BN sample (around $80 \mathrm{~nm}$ thick) on $90 \mathrm{~nm} \mathrm{SiO} 2$ (left) with equivalent DF image of $b$ (right). $c-h$-BN-graphene hetero-structure after transfer of $h$ $B N$ crystal on to target graphene flake (left) with equivalent DF image of c (right). Scale is equivalent for all images, with red circles indicating examples of defect-free regions.

thermal pipette puller to create a taper to $\sim 100 \mu \mathrm{m}$ diameter at the tip. The holder allowed fine wires to be placed inside the pipette from the opposing end to the tapered tip. The pipette could be filled with electrolyte using a syringe and needle. If the tip of the pipette was positioned within a droplet of electrolyte on the graphene electrode surface, contact between the fluid in the pipette and the droplet allowed electrochemical measurements to be recorded. Two wires within the pipette functioned as 
$\mathrm{Ag} / \mathrm{AgCl}$ reference and Pt counter electrodes, with the graphene electrode surface acting as the working electrode. The wires in the pipette needed to be isolated, and therefore a Ag wire with a PTFE insulating coating was used. The two wires were inserted into the pipette, ensuring the length of Pt wire was less than the length of remaining PTFE coating on the $\mathrm{Ag} / \mathrm{AgCl}$ wire to make sure no electrical contact occurred between the two, as shown in Figure S4. The wires were then connected to the reference and counter electrode terminals on the potentiostat (PGSTAT302N, Metrohm Autolab) via metal clips attached to shielded cables.

The graphene device was attached to an $\mathrm{Al}$ coated glass slide as previously described. The glass slide was placed on the working surface of a microscope (up to 100x magnification) and secured. The $\mathrm{Al}$ on the glass slide was connected with a metal clip and shielded cables to the working electrode terminal on the potentiostat. A droplet of the electrolyte was placed on the graphene device surface using a syringe with a fine plastic needle (28 gauge, Microfil, WPI). The syringe was filled with electrolyte and the needle attached, with a small amount of pressure creating a 1-2 mm droplet of electrolyte on the end of the needle. This droplet could be carefully placed on the graphene device, positioned so that the trench in the Si filled with electrolyte and made contact with the graphene surface. The hydrophobic HMDS layer on the device substrate ensured the droplet did not significantly wet the $\mathrm{SiN}_{\mathrm{x}}$ surface surrounding the trench and stayed as a roughly hemispherical droplet. The microscope could be used to check that the droplet had filled the trench and was contacting the graphene. Once the electrolyte contacted the graphene, the pipette tip was rapidly positioned into the electrolyte drop with the micromanipulator, ensuring the pipette did not draw up the droplet due to capillary forces. The setup was then ready to record measurements.

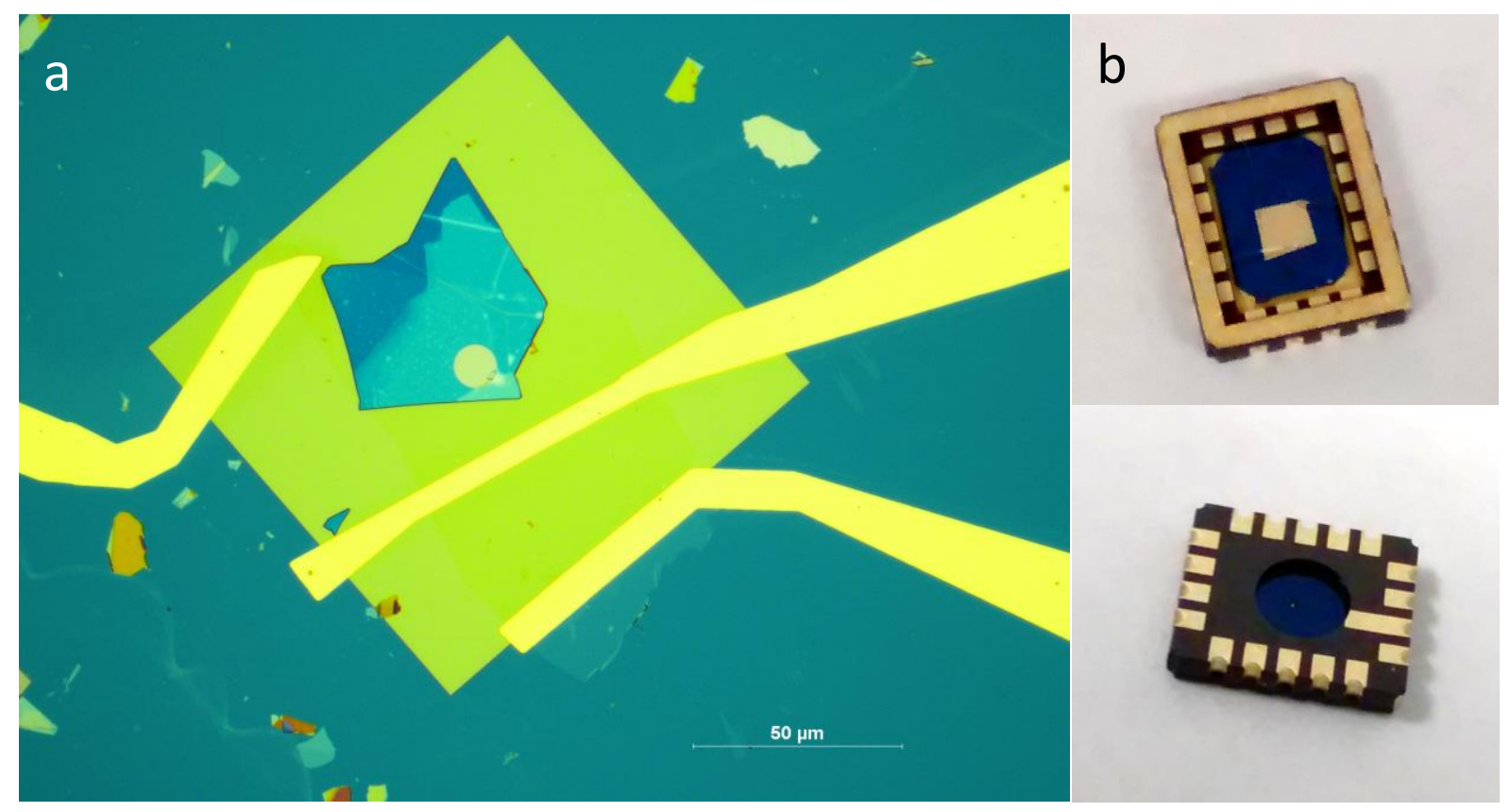

Figure S3. (a) A device demonstrating compatibility with lithographic contact processing. The polymer free nature of the electrochemically active area is conserved, as the lithographic resists are only in contact with the opposing side of the wafer. (b) Images of devices mounted into modified chip carriers. 

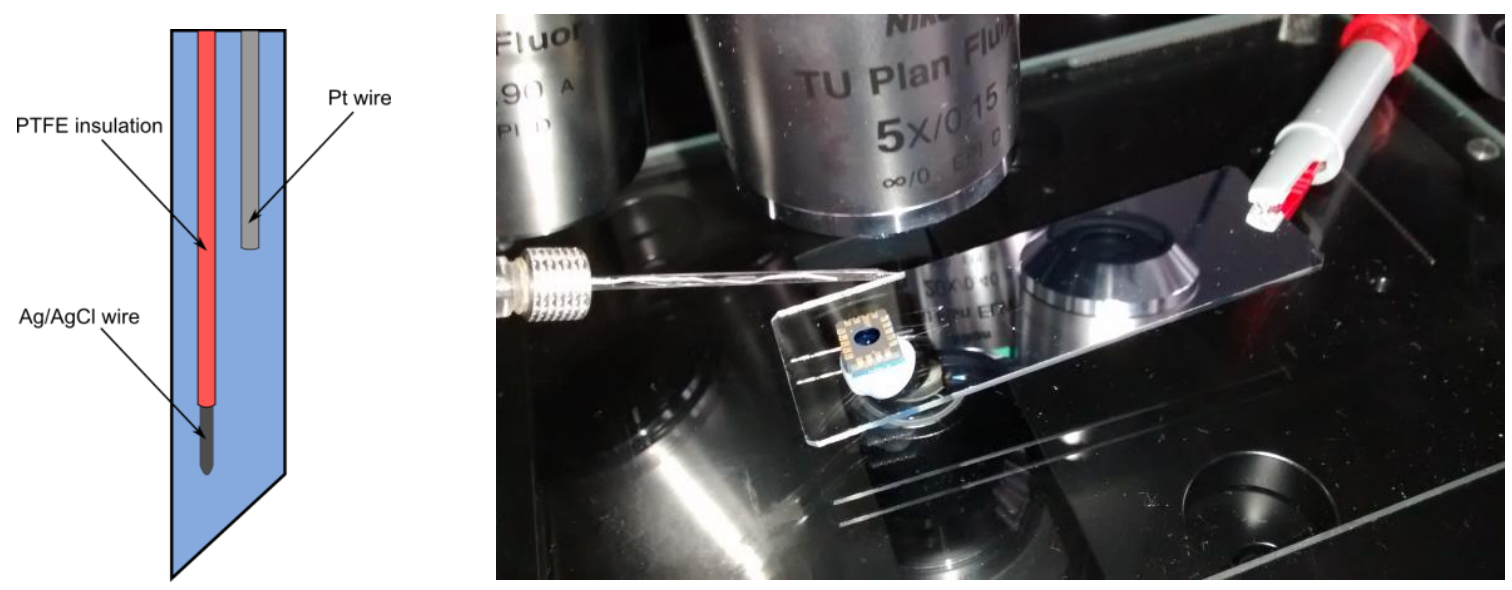

Figure S4 - (a) Schematic diagram of the arrangement of the Ag/AgCl reference and Pt counter electrodes within the pipette holding the measurement electrolyte. (b) Photograph of the graphene device mounted on a glass slide on the measurement stage with microscope.

\section{Control experiments}

To assess whether the device structure was functioning as designed, control measurements were taken. Three control experiments were performed using a slightly modified electrode structure.

A device was fabricated using a $\sim 80 \mathrm{~nm}$ graphite flake in place of the h-BN-graphene structure. Graphite was used as a test conductor to ensure the device would pass a redox current, and could be tested to destruction, avoiding the time consuming transfers required with graphene and h-BN. This device was compared to a device fabricated without a hole in the $\mathrm{SiN}_{\mathrm{x}}$ window or a graphene flake, but with a $\mathrm{Au}$ contact on the underside of the $\mathrm{SiN}_{\mathrm{x}}$ window as with the original device structure. This device still had the etched trench in the $\mathrm{Si}$ and was constructed to test if the $\mathrm{SiN}_{\mathrm{x}}$ was insulating to the electrolyte and no current was passed due to reactions with the Si part of the substrate.

Another device was fabricated with just a h-BN substrate and Au contact with no graphene layer. This was used to access the seal between the $\operatorname{SiN}_{\mathrm{x}}$ substrate and the flake transferred over the hole. This device should pass no redox current as h-BN is insulating, so if significant current was measured electrolyte was likely to be leaking between the h-BN flake and $\mathrm{SiN}_{\mathrm{x}}$ substrate towards to the Au contact at the edges of the h-BN flake. The results of these measurements are shown in Figure S5, which confirms that no leakage occurred through the $\mathrm{SiN}_{\mathrm{x}}$ dielectric as no current was passed between -2 and $1 \mathrm{~V}$. The graphite electrode showed significant current between these potentials, confirming that the conducting layer transferred over the hole allows a redox current to be passed. The h-BN-only test showed a simple capacitance signal with a diffusional component (rectangular box CV with linear offset) as expected. Although no redox mediator is present in the electrolyte, redox behaviour similar to that shown for graphite would be expected between these potentials if the device was leaking as previously described. 

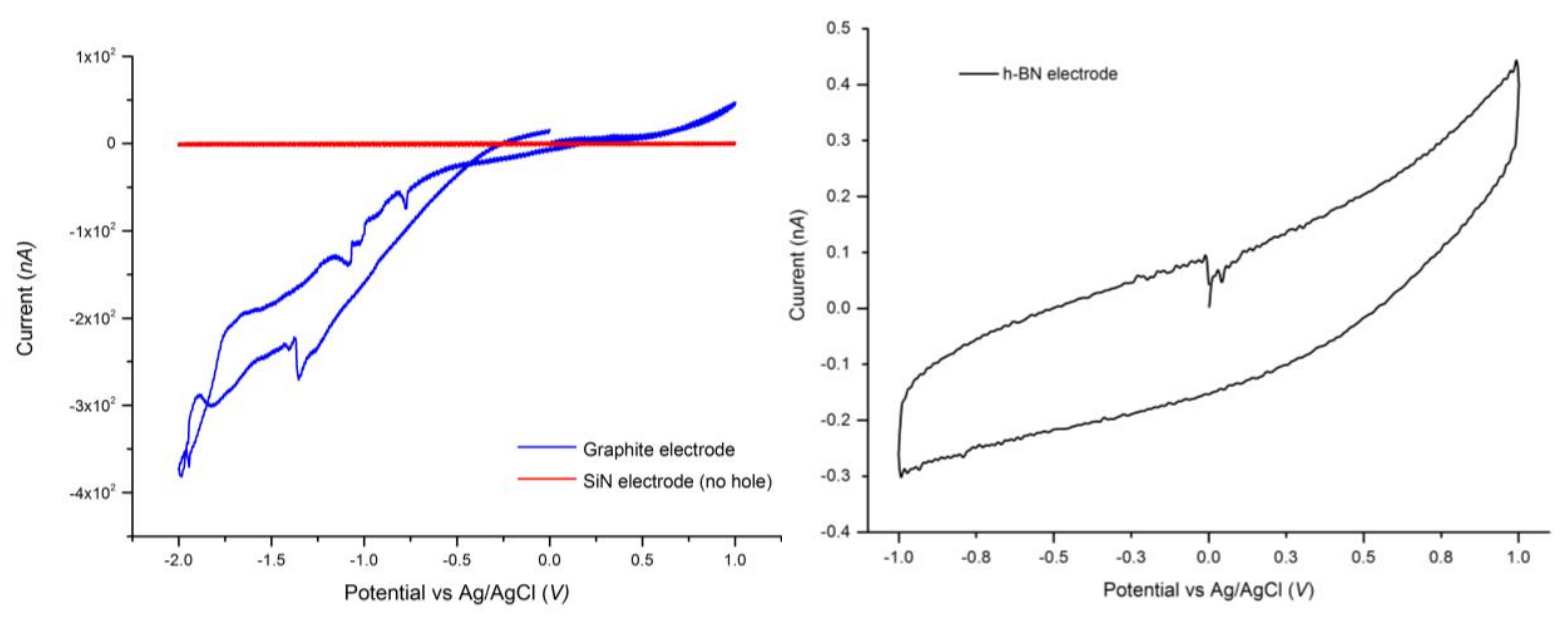

Figure S5 - (a) CVs comparing graphite electrode to SiN electrode (no hole etched in substrate). (b) CV showing response of $h$-BN electrode. In both cases the scan rate was $1 \mathrm{Vs}^{-1}$ and the electrolyte was $1.5 \mathrm{M} \mathrm{KCl}$.

\section{S3. Electrochemical analysis - further information}

\section{Reduction processes at UMEs - formulation}

For a flat, circular UME, the combination of the potential-current relation with hemispherical diffusion of the reactant has non-trivial solutions [3-5], however the key result can be summarised as,

$$
i=\left(4 n F D_{0} C_{O}^{*} r_{0}\right) \cdot f\left(E_{e}\right)=i_{d} \cdot f\left(E_{e}\right)
$$

Where $f\left(E_{e}\right)$ is a sigmoidal-like function describing the transition from zero current to the steady-state current, $i_{d}$.

An empirical solution to this diffusion problem was discovered by Oldham and Zoski, that will predict the response of the function $f\left(E_{e}\right)$ to an accuracy of $0.3 \%$ [6]. Assuming quasi-reversibility, the currentpotential relation can be expressed as,

$$
i=\left(\frac{i_{d}}{\theta}\right) \cdot\left[1+\frac{\pi}{\kappa \theta}\left(\frac{2 \kappa \theta+3 \pi}{4 \kappa \theta+3 \pi^{2}}\right)\right]^{-1}
$$

With,

$$
\begin{gathered}
\kappa=\frac{\pi k^{0} r_{0}}{4 D_{o}} \exp \left[\frac{-\alpha n F}{R T}\left(E-E^{0^{\prime}}\right)\right] \\
\theta=1+\exp \left[\frac{n F}{R T}\left(E-E^{0^{\prime}}\right)\right]
\end{gathered}
$$

This formula assumes Butler-Volmer kinetics apply, the electrode is a flat, circular disc and the diffusion constants are equal for both reactants and products. A further correction to this formula can be added if 
the electrode is recessed into the surrounding substrate. This has the effect of changing the limiting current and function $f\left(E_{e}\right)$ [7]. The effect on $f\left(E_{e}\right)$ is negligible for the recess size in this work $(100 \mathrm{~nm})$.

The effect on the steady state current, $i_{d}$ is given by the factor denoted $I_{c}$,

$$
I_{c}=\frac{4 L}{\pi r_{0}}+1
$$

Where $L$ is the depth of the recess. In this case, for a recess order of $0.1 \mu \mathrm{m}$ and $r_{o}=5 \mu \mathrm{m}$ the correction is $\sim 2.5 \%$. Therefore, it would be sensible to multiply $i_{d}$ by the factor $I_{c}$. Alternative expressions are suggested, derived from simulation, when the ratio of the recess to the electrode radius is $<1[8,9]$, however the basic theoretical form is used for simplicity as the difference between the expressions is small and far more significant sources of error are present in the measurements.

The redox mediator to supporting electrolyte concentration ratio was considered to ensure measurements were not affected by ohmic drop due to insufficient electrolyte concentration and that the redox mediator transport was purely diffusive. The nA amp currents recorded for UMEs mean that Ohmic drop can be considered insignificant [10], with UMEs noted for their ability to record measurements in low conductivity solutions. It was found that $3 \mathrm{mM}$ redox mediator concentration gave sufficient currents to be measured reliably and therefore this was used for measurements. A $150 \mathrm{mM}$ supporting electrolyte is the standard concentration used for biological measurements, which satisfies the condition that the Debye length $(\sim 1 \mathrm{~nm}$ or less) is much smaller than the diffusion layer on experimental timescales, meaning that any non-diffusive transport can be considered negligible [10].

\section{Calibration of formal potential}

The formal potential of the reaction, $E^{0^{\prime}}$, is unknown and needed to be independently estimated. A CV scan was performed using a purchased $3 \mathrm{~mm}$ diameter basal plane HOPG electrode (Cat. No. 002252, IJ Cambria Scientific). This electrode was placed in a glass beaker containing $3 \mathrm{mM} \mathrm{IrCl}_{6}{ }^{2-}$ in $150 \mathrm{mM}$ $\mathrm{KCl}$ supporting electrolyte as with the graphene measurements. Due to the increased electrode area, a departure from the steady-state microelectrode response was seen and two peaks ( $E_{p a}$ and $E_{p c}$ ) consistent with macro-electrodes were measured. An estimation of $E^{0^{\prime}}$ is possible from the average potential of the two peaks $\left(E^{0^{\prime}} \sim\left(E_{p a}-E_{p c}\right) / 2\right)$, assuming $k^{0}$ is not very small (reversible or quasi-reversible kinetics) and the diffusion constants for both oxidised and reduced species are similar [11]. Although $E^{0^{\prime}}$ may be different for HOPG and graphene electrodes, these surfaces are very similar and other studies comparing carbon surfaces in the same electrolyte do not see significant changes in $E^{0^{\prime}}$ between surfaces $[12,13]$. The value of $E^{0^{\prime}}$ obtained from this method was $651 \mathrm{mV}(\mathrm{vs} \mathrm{Ag} / \mathrm{AgCl}$ in $0.15 \mathrm{M} \mathrm{KCl}$ ) and is relatively consistent with other studies using $\mathrm{IrCl}_{6}{ }^{2-}$ on graphene [14], HOPG [15] and Pt [16] electrodes. As the reaction kinetics measured for the graphene UMEs are small $\left(k^{0}=3.04 \pm 0.78 \times 10^{-3} \mathrm{~cm} \mathrm{~s}^{-1}\right)$ the error on the $E^{0^{\prime}}$ estimation only has a weak effect on the values of $k^{0}$. A comparison of the experimental data from the graphene device and HOPG electrode is shown in Figure S6. 


\section{Effect of fitting parameters}

Figure S7 shows how fixing particular fitting parameters $\left(\alpha, r_{0}\right)$ effects the overall quality of fit, and justifies the use of 3 variable fitting parameters. Setting $\alpha$ as 0.5 leads to an overshoot of the experimental data at higher potentials near $E^{0^{\prime}}$ and undershoots the data at lower potentials towards the steady-state limiting current. Therefore it seems necessary to allow $\alpha$ to vary as a fitting parameter to acquire a good (or better) fit.

The effect of setting a fixed value for $r_{o}$ during fitting is particularly detrimental to acquiring a good fit. Although the electrode radius can be directly measured optically, whether this translates directly into

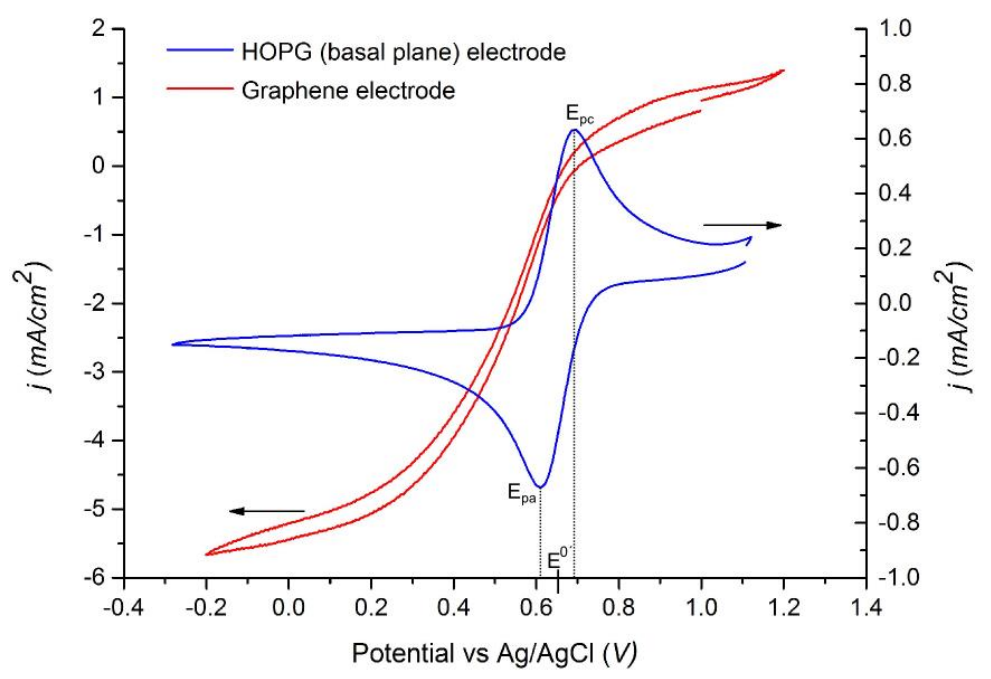

Figure S6 - Comparison of $3 \mathrm{mM}$ $\mathrm{IrCl}_{6}^{2-}$ redox in $150 \mathrm{mM} \mathrm{KCl} \mathrm{supporting}$ electrolyte for graphene UME and HOPG (basal) macro- electrodes. The dotted lines indicate the peaks used to estimate the formal potential, $E^{0^{\prime}}$. Scan rate is $100 \mathrm{mVs}^{-1}$ for both CVs.

the electrochemically active area is less clear and is discussed further in the main text of the paper. If $r_{o}$ is set at the optically measured value, the steady-state limiting current is overestimated by the OldhamZoski formula, suggesting the active electrode area is less than measured optically. As the steady-state limiting current depends on the electrode radius (along with the fixed values of electrolyte concentration, temperature and diffusion constant), the fitting function is forced to tend to the value set by these parameters. This is in turn detrimental to the fitting of the rest of the reduction curve as the values of $\alpha$ and $k^{0}$ become unrealistic in an attempt reach the fixed steady-state current value. By setting $r_{o}$ as a variable fitting parameter the steady-state current magnitude can change allowing far more accurate values of $\alpha$ and $k^{0}$ to be obtained.

The assumed largest source of error in the measured data is the literature value of the diffusion constant, which was not independently measured and can vary with measurement geometries. This error is effectively taken into account as an error in the electrode radius in the fitting procedure and may explain the difference between the optically measured electrode radius and that given by the fit. 


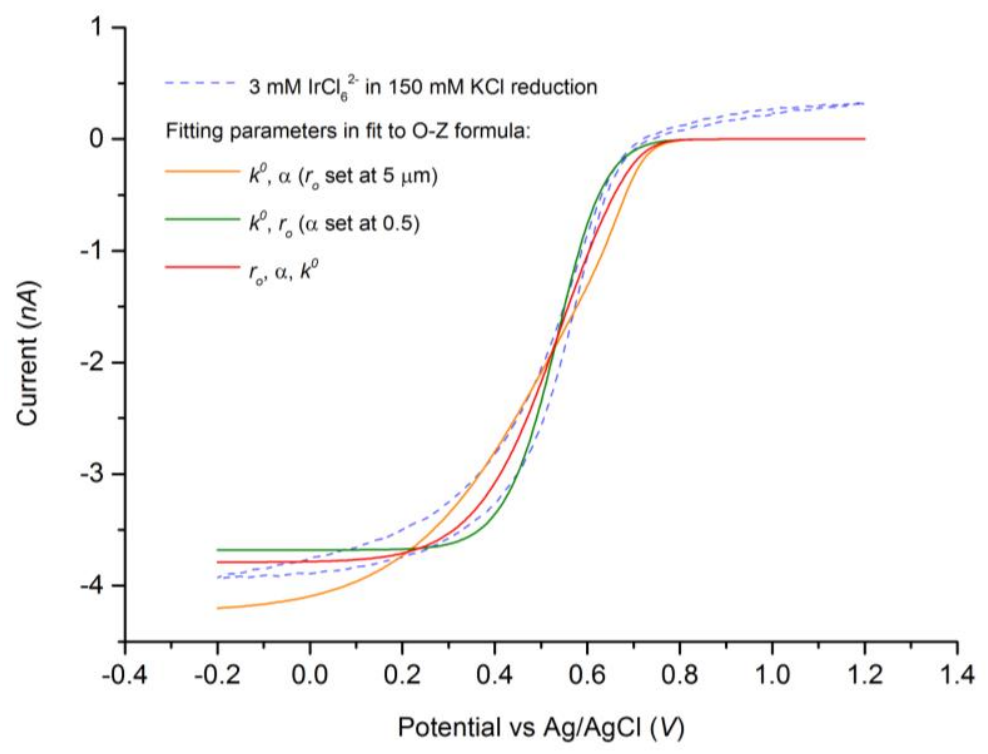

Figure S7 - Comparison of different fitting parameters in Oldham-Zoski formula.

\section{Extended results data set}

Tables S1 and S2 give the full data set for the 4 devices measured with the averaged values.

Table S1 - The parameters $\alpha$ and $k^{0}$ obtained from Oldham-Zoski formula fit to experimental data for the 4 devices. $\sigma$ represents the standard error from the fit (68.3\% confidence level).

\begin{tabular}{|c|c|c|c|c|}
\hline Sample No. & $k^{0}\left(\times 10^{-3} \mathrm{~cm} \mathrm{~s}^{-1}\right)$ & $\pm 2 \sigma\left(\times 10^{-3} \mathrm{~cm} \mathrm{~s}^{-1}\right)($ fit $)$ & $\alpha$ & $\pm 2 \sigma$ (fit) \\
\hline 1 & 3.46 & 0.24 & 0.271 & 0.010 \\
\hline 2 & 4.54 & 0.16 & 0.324 & 0.006 \\
\hline 3 & 1.41 & 0.05 & 0.228 & 0.004 \\
\hline 4 & 2.74 & 0.08 & 0.263 & 0.005 \\
\hline
\end{tabular}

Table $\mathbf{S} \mathbf{2}$ - The mean of $\alpha$ and $k^{0}$ from the 4 devices with the associated error on the mean, where 4 indicates the range of the results $(N=4)$.

\begin{tabular}{|c|c|c|c|}
\hline$\overline{k^{0}}\left(\times 10^{-3} \mathrm{~cm} \mathrm{~s}^{-1}\right)$ & $\pm \Delta k^{0} / \sqrt{N}\left(\times 10^{-3} \mathrm{~cm} \mathrm{~s}^{-1}\right)$ & $\bar{\alpha}$ & $\pm \Delta \alpha / \sqrt{N}$ \\
\hline 3.04 & 0.78 & 0.272 & 0.024 \\
\hline
\end{tabular}

\section{References}

[1] B. Pant, U. Tandon, Etching of silicon nitride in $\mathrm{CCl} 2 \mathrm{~F} 2$, CHF 3, SiF 4, and SF 6 reactive plasma: a comparative study, Plasma chemistry and plasma processing 19(4) (1999) 545-563.

[2] H. Seidel, L. Csepregi, A. Heuberger, H. Baumgärtel, Anisotropic etching of crystalline silicon in alkaline solutions: I. Orientation dependence and behavior of passivation layers, Journal of the electrochemical society 137(11) (1990) 3612.

[3] J.R. Delmastro, D.E. Smith, Methods for obtaining approximate solutions to the expanding-sphere boundaary value problem in direct current polarography, The Journal of Physical Chemistry 71(7) (1967) 2138-2149. 
[4] A.M. Bond, K.B. Oldham, C.G. Zoski, Steady-state voltammetry, Analytica Chimica Acta 216 (1989) $177-230$.

[5] A.M. Bond, K.B. Oldham, C.G. Zoski, Theory of electrochemical processes at an inlaid disc microelectrode under steady-state conditions, Journal of electroanalytical chemistry and interfacial electrochemistry 245(1-2) (1988) 71-104.

[6] K.B. Oldham, C.G. Zoski, Comparison of voltammetric steady states at hemispherical and disc microelectrodes, Journal of Electroanalytical Chemistry and Interfacial Electrochemistry 256(1) (1988) 11-19.

[7] A.M. Bond, D. Luscombe, K.D. Oldham, C.G. Zoski, Comparison of the Chronoamperometric Response At Inland and Recessed Disc Microelectrodes, Journal of electroanalytical chemistry and interfacial electrochemistry 249(1-2) (1988) 35-62.

[8] J. Guo, E. Lindner, Cyclic voltammetry at shallow recessed microdisc electrode: Theoretical and experimental study, Journal of Electroanalytical Chemistry 629(1-2) (2009) 180-184.

[9] P. Bartlett, S. Taylor, An accurate microdisc simulation model for recessed microdisc electrodes, Journal of Electroanalytical Chemistry 453(1-2) (1998) 49-60.

[10] E.J. Dickinson, J.G. Limon-Petersen, N.V. Rees, R.G. Compton, How much supporting electrolyte is required to make a cyclic voltammetry experiment quantitatively "diffusional"? A theoretical and experimental investigation, The Journal of Physical Chemistry C 113(25) (2009) 11157-11171.

[11] A.J. Bard, L.R. Faulkner, Electrochemical Methods: Fundamentals and applications, (2001).

[12] M. Velický, M.A. Bissett, P.S. Toth, H.V. Patten, S.D. Worrall, A.N. Rodgers, E.W. Hill, I.A. Kinloch, K.S. Novoselov, T. Georgiou, Electron transfer kinetics on natural crystals of MoS 2 and graphite, Physical Chemistry Chemical Physics 17(27) (2015) 17844-17853.

[13] K.R. Kneten, R.L. McCreery, Effects of redox system structure on electron-transfer kinetics at ordered graphite and glassy carbon electrodes, Analytical Chemistry 64(21) (1992) 2518-2524.

[14] M. Velický, D.F. Bradley, A.J. Cooper, E.W. Hill, I.A. Kinloch, A. Mishchenko, K.S. Novoselov, H.V. Patten, P.S. Toth, A.T. Valota, S.D. Worrall, R.A.W. Dryfe, Electron Transfer Kinetics on Monoand Multilayer Graphene, ACS Nano 8(10) (2014) 10089-10100.

[15] G. Zhang, A.S. Cuharuc, A.G. Güell, P.R. Unwin, Electrochemistry at highly oriented pyrolytic graphite (HOPG): lower limit for the kinetics of outer-sphere redox processes and general implications for electron transfer models, Physical Chemistry Chemical Physics 17(17) (2015) 11827-11838.

[16] C. Beriet, D. Pletcher, A further microelectrode study of the influence of electrolyte concentration on the kinetics of redox couples, Journal of Electroanalytical Chemistry 375(1) (1994) 213-218. 